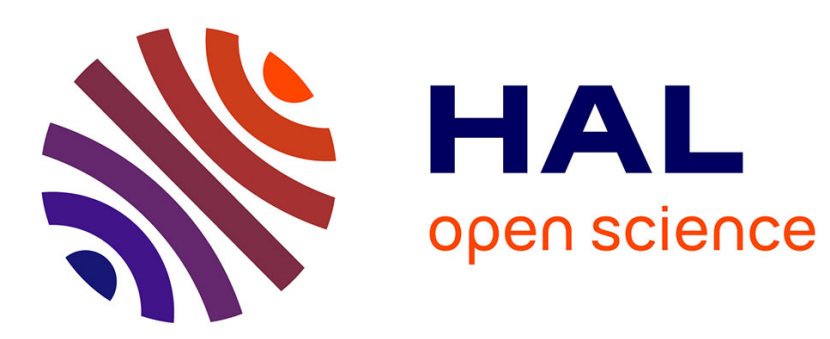

\title{
Weak existence and uniqueness for affine stochastic Volterra equations with L1-kernels
}

Eduardo Abi Jaber

\section{To cite this version:}

Eduardo Abi Jaber. Weak existence and uniqueness for affine stochastic Volterra equations with L1-kernels. Bernoulli, 2021, 27 (3), pp.1583-1615. 10.3150/20-BEJ1284 . hal-02412741v2

HAL Id: hal-02412741

https://hal.science/hal-02412741v2

Submitted on 18 Jun 2020

HAL is a multi-disciplinary open access archive for the deposit and dissemination of scientific research documents, whether they are published or not. The documents may come from teaching and research institutions in France or abroad, or from public or private research centers.
L'archive ouverte pluridisciplinaire HAL, est destinée au dépôt et à la diffusion de documents scientifiques de niveau recherche, publiés ou non, émanant des établissements d'enseignement et de recherche français ou étrangers, des laboratoires publics ou privés. 


\title{
Weak existence and uniqueness for affine stochastic Volterra equations with $L^{1}$-kernels
}

\author{
Eduardo Abi Jaber*
}

June 18,2020

\begin{abstract}
We provide existence, uniqueness and stability results for affine stochastic Volterra equations with $L^{1}$-kernels and jumps. Such equations arise as scaling limits of branching processes in population genetics and self-exciting Hawkes processes in mathematical finance. The strategy we adopt for the existence part is based on approximations using stochastic Volterra equations with $L^{2}$-kernels combined with a general stability result. Most importantly, we establish weak uniqueness using a duality argument on the Fourier-Laplace transform via a deterministic Riccati-Volterra integral equation. We illustrate the applicability of our results on Hawkes processes and a class of hyperrough Volterra Heston models with a Hurst index $H \in(-1 / 2,1 / 2]$.
\end{abstract}

Keywords: Stochastic Volterra equations, Affine Volterra processes, Riccati-Volterra equations, superprocesses, Hawkes processes, rough volatility.

MSC2010 Classification: 60H20, 60G22, 45D05

\section{Introduction}

We establish weak existence, uniqueness and stability results for stochastic Volterra equation with locally $L^{1}$-kernels $K$ in the form

$$
X_{t}=G_{0}(t)+\int_{0}^{t} K(t-s) Z_{s} d s, \quad t \geq 0
$$

*Université Paris 1 Panthéon-Sorbonne, Centre d'Economie de la Sorbonne, 106, Boulevard de l'Hôpital, 75013 Paris, eduardo.abi-jaber@univ-paris1.fr. I would like to thank Mathieu Rosenbaum for presenting me the uniqueness problem, and Ryan McCrickerd for interesting discussions. I am also grateful for the editor and two anonymous referees whose insightful comments and suggestions have significantly improved the manuscript. 
for a given function $G_{0}: \mathbb{R}_{+} \rightarrow \mathbb{R}$ where $Z$ is a real-valued semimartingale, starting from zero, with affine characteristics in $X$

$$
(b X, c X, \nu(d \zeta) X)
$$

with $b \in \mathbb{R}, c \geq 0, \nu$ a nonnegative measure on $\mathbb{R}_{+}$such that $\int_{\mathbb{R}_{+}} \zeta^{2} \nu(d \zeta)<\infty$, with respect to the 'truncation function' $\chi(\zeta)=\zeta$. For $L^{2}$-kernels this formulation was recently introduced in Abi Jaber et al. (2019a), where $Z$ is a semimartingale but whose characteristics are absolutely continuous with respect to the Lebesgue measure. In the $L^{1}$ setting, $X$ may fail to be absolutely continuous with respect to the Lebesgue measure, as will be explained in the sequel. For this reason, our study falls beyond the scope of Abi Jaber et al. (2019a).

Our motivation for studying such convolution equations is twofold. Stochastic Volterra equations with kernels that are locally in $L^{1}$ but not in $L^{2}$ with $c>0 \nu \equiv 0$ arise as scaling limits of branching processes in population genetics and self-exciting Hawkes processes in mathematical finance. As we will highlight in the sequel, the $L^{1}$-framework allows for instance to make sense of fractional dynamics, inspired by the fractional Brownian motion, for negative Hurst indices $H \in(-1 / 2,0)$.

- From branching processes to stochastic Volterra equations. The link was formulated for the first time in Mytnik and Salisbury (2015) to motivate the study of stochastic Volterra equations with $L^{2}$-kernels. In the sequel we re-formulate the aforementioned introductory exposition linking super-processes with stochastic Volterra equations with $L^{1}$ kernels. Consider a system of $n$ reactant particles in one dimension moving independently according to a standard Brownian motion and branching only in the presence of a catalyst. The catalyst region at a certain time $t$ is defined as the support of some deterministic measure $\rho_{t}(d x)$. Whenever a particle enters in the catalyst region and after spending a random time in the vicinity of the catalyst, it will either die or split into two new particles, with equal probabilities. The measure $\rho_{t}(d x)$ determines the local branching rate in space and time depending on the location and the concentration of the catalyst. Two typical examples are $\rho_{t}(d x) \equiv \bar{\rho} d x$ where the branching occurs in the entire space with constant rate $\bar{\rho}$ and $\rho_{t}(d x)=\delta_{0}(d x)$ for a branching occurring with infinite rate only when the particle hits a highly concentrated single point catalyst located at 0 . In case of branching, the two offspring particles evolve independently with the same spatial movement and branching mechanism as their parent.

One can view the reactant as a rescaled measure-valued process $\left(\bar{Y}_{t}^{n}(d x)\right)_{t \geq 0}$ defined by

$$
\bar{Y}_{t}^{n}(B)=\frac{\text { number of particles in } B \text { at time } t}{n}, \text { for every Borel set } B .
$$

Sending the number of particles to infinity, one can establish the convergence towards a measure-valued macroscopic reactant $\bar{Y}$, coined catalytic super-Brownian motion, which solves an infinite dimensional martingale problem, see Dawson and Fleischmann (1991); 
Etheridge (2000); Perkins (2002) and the references therein. Moreover, in the presence of a suitable deterministic catalyst $\rho=\left(\rho_{t}(d x)\right)_{t \geq 0}$ having no atoms, the measure-valued process $\bar{Y}$ admits a density $\bar{Y}_{t}(d x)=Y_{t}(x) d x$ solution to the following stochastic partial differential equation in mild form

$$
Y_{t}(x)=\int_{\mathbb{R}} p_{t}(x-y) Y_{0}(y) d y+\int_{[0, t] \times \mathbb{R}} p_{t-s}(x-y) \sqrt{Y_{s}(y)} W^{\rho}(d s, d y) .
$$

where $Y_{0}$ is an input curve, $p_{t}(x)=(2 \pi t)^{-1 / 2} \exp \left(-x^{2} /(2 t)\right)$ is the heat kernel and $W^{\rho}$ is a space-time noise with covariance structure determined by $\rho$, we refer to Zähle (2005) for more details. The previous equation is only valid if $\rho$ has no atoms. One could still heuristically set $\rho_{t}(d x)=\delta_{0}(d x)$ in (1.3) for the extreme case of a single point catalyst at 0 , which would formally correspond to the catalytic super-Brownian motion of Dawson and Fleischmann (1994). Then, the space-time noise reduces to a standard Brownian motion $W$ so that evaluation at $x=0$ yields

$$
Y_{t}(0)=g_{0}(t)+\frac{1}{\sqrt{2 \pi}} \int_{0}^{t}(t-s)^{-1 / 2} d Z_{s}
$$

where $d Z_{t}=\sqrt{Y_{t}(0)} d W_{t}$ and $g_{0}(t)=\int_{\mathbb{R}} p_{t}(y) Y_{0}(y) d y$. The link with stochastic Volterra equations of the form (1.1) is established by considering the local occupation time at the catalyst point 0 defined by

$$
X_{t}=\lim _{\varepsilon \rightarrow 0} \int_{0}^{t} \int_{\mathbb{R}} p^{\varepsilon}(y) \bar{Y}_{s}(d y) d s, \quad t \geq 0,
$$

where $p^{\varepsilon}$ is a suitable smoothing kernel of the dirac point mass at 0 . Integrating both sides of equation (1.4) with respect to time and formally interchanging the integrals lead to

$$
\begin{aligned}
X_{t} & =\int_{0}^{t} Y_{s}(0) d s \\
& =\int_{0}^{t} g_{0}(s) d s+\frac{1}{\sqrt{2 \pi}} \int_{0}^{t}(t-s)^{-1 / 2} Z_{s} d s,
\end{aligned}
$$

such that $Z$ is a continuous semimartingale with affine characteristics $(0, X, 0)$. Consequently, $X$ solves (1.1) for the kernel

$$
K_{0}(t)=\frac{t^{-1 / 2}}{\sqrt{2 \pi}}, \quad t>0,
$$

which is locally in $L^{1}$ but not in $L^{2}$. Needless to say, one is not allowed to plug the Dirac measure in (1.3). Indeed, in the presence of a single point catalyst, the catalytic super-Brownian motion does not admit a density at the catalyst position as shown by 
Dawson and Fleischmann (1994) and the identities (1.3) and (1.6) break down. The local occupation time $X$ is even singular with respect to the Lebesgue measure, see Dawson et al. (1995); Fleischmann and Le Gall (1995). Still, one can rigorously prove that the local occupation time $X$ defined by (1.5) solves (1.7) by appealing to the martingale problem of the measure-valued process $\bar{Y}$, we refer to Appendix A for a rigorous derivation.

- From Hawkes processes to stochastic Volterra equations. More recently, for particular choices of $G_{0}$ and kernels, solutions to (1.1) were obtained in Jusselin and Rosenbaum (2018) as scaling limits of Hawkes processes $\left(N^{n}\right)_{n \geq 1}$ with respective intensities

$$
\lambda_{t}^{n}=g_{0}^{n}(t)+\int_{0}^{t} K^{n}(t-s) d N_{s}^{n}, \quad t \geq 0
$$

for some suitable function $g_{0}^{n}$ and kernel $K^{n}$. The rescaled sequence of integrated accelerated intensities $X^{n}=\frac{1}{n} \int_{0}^{\cdot} \lambda_{n s}^{n} d s$ is shown to converge to a continuous process $X$ satisfying (1.1) for the fractional kernel ${ }^{1}$

$$
K_{H}(t)=\frac{t^{H-1 / 2}}{\Gamma(H+1 / 2)}, \quad t>0
$$

with $H \in(1 / 2,1 / 2]$. We note that for $H=0$ the fractional kernel reduces to (1.8), up to a normalizing constant. In other words, when $H=0$, the scaling limit of the integrated intensities of Hawkes processes can be seen as the local occupation time of the catalytic super-Brownian motion of Dawson and Fleischmann (1994), provided uniqueness holds. Similarly, when $H \leq 0, K_{H}$ lies locally in $L^{1}$ but not in $L^{2}$, and one can also show that in this case $X$ is not absolutely continuous with respect to the Lebesgue measure, see Jusselin and Rosenbaum (2018, Proposition 4.6). For $H>0$, the kernel (1.9) is nothing else but the kernel that appears in the celebrated Mandelbrot and Van Ness (1968) decomposition of fractional Brownian motion $W^{H}$ :

$$
W_{t}^{H}=\int_{0}^{t} K_{H}(t-s) d W_{s}+\int_{-\infty}^{0}\left(K_{H}(t-s)-K_{H}(-s)\right) d W_{s}
$$

where $W$ is a two-sided standard Brownian motion and $H>0$ is required to make sense of the stochastic convolution with respect to Brownian motion in the $L^{2}$-theory of Kiyosi Itô. In this sense, the $L^{1}$-framework allows for a generalization of fractional type dynamics with negative Hurst indices.

In both of the motivating cases, one can compute the Laplace transform of $X$, modulo a deterministic Riccati-Volterra equation of the form

$$
\psi(t)=\int_{0}^{t} K_{H}(t-s)\left(\frac{1}{2} \psi^{2}(s)-1\right) d s
$$

\footnotetext{
${ }^{1}$ To be more precise, in Jusselin and Rosenbaum (2018), the limiting kernel is not the fractional kernel but the so-called Mittag-Leffler function and the process $Z$ there has characteristics $(0, X, 0)$. This can be equivalently re-written with the fractional kernel modulo the addition of a suitable drift $b X$, we refer to Example 2.10 below for more details.
} 
either by using the dual process of the catalytic super-Brownian motion, see Dawson and Fleischmann (1994, Equations (4.2.1)-(4.2.2)), or by exploiting the affine structure of the approximating Hawkes processes, see Jusselin and Rosenbaum (2018, Theorem 3.4). Both constructions provide solutions to (1.1), but do not yield uniqueness. Establishing weak uniqueness is one of the main motivation of this work.

In the present paper, we provide a generic treatment of the limiting macroscopic equation (1.1) and we allow for (infinite activity) jumps in $Z$. For instance, Hawkes processes can be recovered by setting $c=0$ and $\nu=\delta_{1}$. The strategy we adopt is based on approximations using stochastic Volterra equations with $L^{2}$ kernels, whose existence and uniqueness theory is now well-established, see Abi Jaber et al. (2019a,b) and the references therein. By doing so, we avoid the infinite-dimensional analysis used for super-processes, we also circumvent the need to study scaling limits of Hawkes processes, allowing for more generality in the choice of kernels $K$ and input functions $G_{0}$. Along the way, we derive a general stability result that encompasses the motivating example with Hawkes processes. Most importantly, we establish weak uniqueness using a duality argument on the Fourier-Laplace transform of $X$ via a deterministic Riccati-Volterra integral equation. In particular, this expression extends the one obtained for affine Volterra processes with $L^{2}$-kernels in Abi Jaber et al. (2019b); Cuchiero and Teichmann (2020). We illustrate the applicability of our results on a class of hyper-rough Volterra Heston models with a Hurst index $H \in(-1 / 2,1 / 2]$ and jumps complementing the results of Abi Jaber et al. (2019b); El Euch and Rosenbaum (2019); Jusselin and Rosenbaum (2018). Such models have recently known a growing interest to account for rough volatility, a universal phenomena observed in financial markets, see Gatheral et al. (2018).

Notations $\Delta_{h}$ stands for the shift operator, i.e. $\Delta_{h} g=g(h+\cdot)$ and $d g$ is the distributional derivative of a right-continuous function $g$ with locally bounded variation, i.e. $d g((s, t])=g(t)-g(s)$. For a suitable Borel function $f$ the quantity $\int_{0}^{\cdot} f(s) d g(s)$ will stand for the Lebesgue-Stieltjes integral, whenever the integral exists. Similarly, for each $t<T$, the convolution $\int_{0}^{t} f(t-s) d g(s)$ is defined as the Lebesgue-Stieltjes integral $\int_{0}^{T} \mathbf{1}_{[0, t]} f(t-s) d g(s)$ whenever this latter quantity is well-defined.

Outline Section 2 states our main existence, uniqueness and stability results together with the expression for the Fourier-Laplace transform. Section 3 provides a-priori estimates for the solution. In Section 4, we derive a general stability results for stochastic Volterra equations with $L^{1}$-kernels. These results are used to establish weak existence for the stochastic Volterra equation in Section 5. Furthermore, an existence result for RiccatiVolterra equations with $L^{1}$-kernels is derived there. Weak uniqueness is then established by completely characterizing the Fourier-Laplace transform of the solution in terms of the Riccati-Volterra equation of Section 6. In Section 7, we apply our results to obtain existence, uniqueness and the characteristic function of the log-price in hyper-rough Volterra Heston models. Finally, we provide a more rigorous derivation of the stochastic Volterra equation satisfied by the local occupation time of the catalytic super-Brownian motion in 
Appendix A.

\section{Main results}

In this section, we present our main results together with the strategy we adopt. We start by making precise the concept of solution.

We call $X$ a weak solution to (1.1) for the input $\left(G_{0}, K, b, c, \nu\right)$, if there exists a filtered probability space $\left(\Omega, \mathcal{F},\left(\mathcal{F}_{t}\right)_{t \geq 0}, \mathbb{P}\right)$ supporting a non-decreasing, nonnegative, continuous and adapted process $X$ and a semimartingale $Z$ whose characteristics are given by (1.2) such that (1.1) holds $\mathbb{P}$-almost surely. We stress that a weak solution is given by the triplet $\left(X,\left(\Omega, \mathcal{F},\left(\mathcal{F}_{t}\right)_{t \geq 0}, \mathbb{P}\right), Z\right)$. To ease notations we simply denote the solution by $X$. In this case, $Z$ admits the following decomposition

$$
Z_{t}=b X_{t}+M_{t}^{c}+M_{t}^{d}, \quad t \geq 0
$$

where $M^{c}$ is a continuous local martingale of quadratic variation $c X$ and $M^{d}$ is a purely discontinuous local martingale given by $\int_{[0, t] \times \mathbb{R}_{+}} \zeta\left(\mu^{Z}(d t, d \zeta)-\nu(d \zeta) d X_{t}\right)$ and $\mu^{Z}$ is the jump measure of $Z$, we refer to Jacod and Shiryaev (2003, II.2.5-6). We say that weak uniqueness holds for the inputs $\left(G_{0}, K, b, c, \nu\right)$ if given two weak solutions $\left(X,\left(\Omega, \mathcal{F},\left(\mathcal{F}_{t}\right)_{t \geq 0}, \mathbb{P}\right), Z\right)$ and $\left(X^{\prime},\left(\Omega^{\prime}, \mathcal{F}^{\prime},\left(\mathcal{F}_{t}^{\prime}\right)_{t \geq 0}, \mathbb{P}^{\prime}\right), Z^{\prime}\right), X$ and $X^{\prime}$ have the same finite dimensional marginals.

One first notes that the formulation (1.1), when $\nu \equiv 0$, differs from the one given in Jusselin and Rosenbaum (2018, Equation (3)), where

$$
X_{t}=G_{0}(t)+\int_{0}^{t}\left(\int_{0}^{t-s} K(r) d r\right) d Z_{s}
$$

Although these two formulations are equivalent, thanks to a stochastic Fubini theorem, the advantages of considering the formulation (1.1) as starting point, which is inspired by the 'martingale problem' formulation of stochastic Volterra equations recently introduced in Abi Jaber et al. (2019a) will become clear in the sequel.

The following lemma establishes the link with stochastic Volterra equations with $L^{2}$ kernels, as the one studied for instance in Abi Jaber et al. (2019a,b); Cuchiero and Teichmann (2020).

Lemma 2.1. Fix $K \in L_{\text {loc }}^{2}\left(\mathbb{R}_{+}, \mathbb{R}\right)$ and $g_{0} \in L_{\text {loc }}^{1}\left(\mathbb{R}_{+}, \mathbb{R}\right)$. Assume that there exists a nondecreasing continuous adapted process $X$ on some filtered probability space $\left(\Omega, \mathcal{F},\left(\mathcal{F}_{t}\right)_{t \geq 0}, \mathbb{P}\right)$ such that

$$
X_{t}=\int_{0}^{t} g_{0}(s) d s+\int_{0}^{t} K(t-s) Z_{s} d s
$$


with $Z$ given by (2.1). Then, $X=\int_{0}^{\cdot} Y_{s} d s$ where $Y$ is a nonnegative weak solution to the following stochastic Volterra equation

$$
Y_{t}=g_{0}(t)+\int_{0}^{t} K(t-s) d Z_{s}, \quad \mathbb{P} \otimes d t-a . e .
$$

where the differential characteristics of $Z$ with respect to the Lebesgue measure are given by $(b Y, c Y, \nu(d \zeta) Y)$.

Conversely, assume there exists a nonnegative weak solution $Y$ to the stochastic Volterra equation (2.3) such that $\int_{0}^{T} Y_{s} d s<\infty$ for all $T>0$, then $X=\int_{0}^{\cdot} Y_{s} d s$ is a continuous non-decreasing solution to (2.2).

Proof. Fix $t \geq 0$. An application of a stochastic Fubini theorem, see Abi Jaber et al. (2019a, Lemma 3.2), yields

$$
\begin{aligned}
\int_{0}^{t} K(t-s) Z_{s} d s & =\int_{0}^{t} K(s)\left(\int_{0}^{t-s} d Z_{s}\right) d s \\
& =\int_{0}^{t}\left(\int_{0}^{t-r} K(s) d s\right) d Z_{r} \\
& =\int_{0}^{t}\left(\int_{0}^{t} K(s-r) \mathbf{1}_{\{r \leq s\}} d s\right) d Z_{r} \\
& =\int_{0}^{t}\left(\int_{0}^{s} K(s-r) d Z_{r}\right) d s
\end{aligned}
$$

Thus, $X$ admits a density $Y$ with respect to the Lebesgue measure, such that

$$
Y_{t}=g_{0}(t)+\int_{0}^{t} K(t-r) d Z_{r}
$$

and the characteristics of $Z$ read

$$
\left(b \int_{0}^{r} Y_{s} d s, c \int_{0}^{\cdot} Y_{s} d s, \int_{[0, \cdot] \times \mathbb{R}_{+}} Y_{s} d s \nu(d \zeta)\right) .
$$

Since $X$ is non-decreasing almost surely, $Y$ is nonnegative $\mathbb{P} \otimes d t$. The claimed stochastic Volterra equation (2.3) readily follows. The converse direction follows along the same lines by integrating both sides of (2.3) and applying a stochastic Fubini theorem as above to get (2.2).

\subsection{Uniqueness and Fourier-Laplace transform}

We start by stating our first main result concerning the representation of the FourierLaplace transform of the joint process $\left(X, M^{c}, M^{d}\right)$ and the weak uniqueness statement for 
(1.1) in terms of a solution to the Riccati-Volterra equation

$$
\begin{aligned}
\psi(t)= & \int_{0}^{t} K(t-s) F(s, \psi(s)) d s \\
F(s, u)= & f_{0}(s)+\frac{1}{2} c f_{1}^{2}(s)+\left(b+c f_{1}(s)\right) u+\frac{c}{2} u^{2} \\
& \quad+\int_{\mathbb{R}_{+}}\left(e^{\left(f_{2}(s)+u\right) \zeta}-1-\left(f_{2}(s)+u\right) \zeta\right) \nu(d \zeta),
\end{aligned}
$$

where $f_{0}, f_{1}, f_{2}: \mathbb{R}_{+} \mapsto \mathbb{B}$ are suitable functions. We introduce the following process which enters in the representation of the Fourier-Laplace transform:

$$
\begin{array}{rlrl}
G_{t}(s) & =G_{0}(s)+\int_{t}^{s} g_{t}(u) d u, & t \leq s, \\
g_{t}(u) & =\int_{0}^{t} K(u-r) d Z_{r}, & & t<u .
\end{array}
$$

The stochastic convolution $\int_{0}^{t} K(s-r) d Z_{r}=\int_{0}^{t} \Delta_{s-t} K(t-r) d Z_{r}$ is well-defined as an Itô integral, for all $s>t$, provided that the shifted kernels $\Delta_{h} K:=K(\cdot+h)$ are in $L_{\text {loc }}^{2}\left(\mathbb{R}_{+}, \mathbb{R}\right)$ for any $h>0$.

Theorem 2.2. Fix $b \in \mathbb{R}, c \geq 0$ and $\nu$ a nonnegative measure supported on $\mathbb{R}_{+}$such that $\int_{\mathbb{R}_{+}} \zeta^{2} \nu(d \zeta)<\infty$. Fix a kernel $K \in L_{\text {loc }}^{1}\left(\mathbb{R}_{+}, \mathbb{R}\right)$ such that its shifted kernels $\Delta_{h} K=$ $K(\cdot+h)$ are in $L_{\text {loc }}^{2}\left(\mathbb{R}_{+}, \mathbb{R}\right)$ for any $h>0$, and a non-decreasing continuous function $G_{0}: \mathbb{R}_{+} \mapsto \mathbb{R}$. Fix $T \geq 0$ and three continuous functions $f_{0}, f_{1}, f_{2}:[0, T] \mapsto \mathbb{C}$. Assume that there exists a solution $\psi \in C([0, T], \mathbb{C})$ to the Riccati-Volterra equation (2.4)-(2.5) such that

$$
\sup _{t \leq T} \int_{\mathbb{R}_{+}} e^{\Re\left(f_{2}(t)+\psi(t)\right) \zeta} \zeta^{2} \nu(d \zeta)<\infty
$$

Then, for any weak solution $X$ of (1.1), the joint conditional Fourier-Laplace transform of

$$
R_{t, T}=\int_{t}^{T} f_{0}(T-s) d X_{s}+\int_{t}^{T} f_{1}(T-s) d M_{s}^{c}+\int_{t}^{T} f_{2}(T-s) d M_{s}^{d},
$$

where $M^{c}$ and $M^{d}$ are the local martingales appearing in (2.1), is given by

$$
\mathbb{E}\left[\exp \left(R_{t, T}\right) \mid \mathcal{F}_{t}\right]=\exp \left(\int_{t}^{T} F(T-s, \psi(T-s)) d G_{t}(s)\right), \quad t \leq T,
$$

where $G_{t}$ is defined as in (2.6). In particular, if the Riccati-Volterra equation (2.4)-(2.5) with $f_{2}=f_{3} \equiv 0$, admits a continuous solution $\psi$ such that $\sup _{t \leq T} \int_{\mathbb{R}_{+}} e^{\Re(\psi(t)) \zeta} \zeta^{2} \nu(d \zeta)<$ $\infty$ for any continuous function $f_{0}:[0, T] \mapsto i \mathbb{R}$ and any $T \geq 0$, then weak uniqueness holds for (1.1) for the input $\left(G_{0}, K, b, c, \nu\right)$. 
Proof. The proof is detailed in Section 6.

The following example illustrates the applicability of Theorem 2.2 in the case of pure jump Hawkes processes. The example will be continued in Example 2.10 to illustrate the scaling limits of Hawkes processes mentioned in the introduction. Section 7 provides another example of application of Theorem 2.2 in the context of rough volatility modeling.

Example 2.3. Let $N$ denote a counting process with instantaneous intensity

$$
\lambda_{t}=g_{0}(s)+\int_{0}^{t} K(t-s) d N_{s}
$$

for some $g_{0}, K \in L_{\text {loc }}^{1}\left(\mathbb{R}_{+}, \mathbb{R}_{+}\right)$. We are interested in the computation of the joint FourierLaplace transform of $(\lambda, N)$, more precisely of the quantity:

$$
\int_{0}^{T} h_{0}(T-s) \lambda_{s} d s+\int_{0}^{T} h_{2}(T-s) d N_{s},
$$

for some continuous functions $h_{0}, h_{2}: \mathbb{R}_{+} \mapsto \mathbb{C}$. By an application of Lemma 2.1, the integrated intensity $X=\int_{0}^{\cdot} \lambda_{s} d s$ solves (1.1) with $G_{0}=\int_{0}^{\cdot} g_{0}(s) d s$ and $Z=N$ with affine characteristics in (1.2) given by

$$
\left(X, 0, \delta_{1}(d \zeta) X\right)
$$

meaning that $(b, c, \nu)=\left(1,0, \delta_{1}\right)$ and $M^{d}=(N-X)$. Under the assumptions of Theorem 2.2, setting $f_{0}=h_{0}-h_{2}, f_{1} \equiv 0$ and $f_{2}=h_{2}$ the joint Fourier-Laplace transform of $(\Lambda, N)$ is given by

$\mathbb{E}\left[\exp \left(\int_{0}^{T} h_{0}(T-s) \lambda_{s} d s+\int_{0}^{T} h_{2}(T-s) d N_{s}\right)\right]=\exp \left(\int_{0}^{T} F(T-s, \psi(T-s)) g_{0}(s) d s\right)$

where the Riccati-Volterra equations (2.4)-(2.5) read

$$
\begin{aligned}
\psi(t) & =\int_{0}^{t} K(t-s) F(s, \psi(s)) d s, \quad t \leq T, \\
F(s, u) & =h_{0}(s)+e^{\left(h_{2}(s)+u\right)}-1 .
\end{aligned}
$$

We refer to Theorem 2.5 and Remark 2.6 below for the existence of such $\psi$. In particular, setting $h_{0} \equiv 0$ and $h_{2} \equiv i a$ for some $a \in \mathbb{R}$, we recover the formula of Hawkes and Oakes (1974, Theorem 2) derived using cluster representations of Hawkes processes, see also El Euch and Rosenbaum (2019, Theorem 3.1).

Under additional assumptions on $K$ we prove the existence of a solution to the RiccatiVolterra equations (2.4)-(2.5). For this we recall the notion of the resolvent of the first 
kind of a kernel: a measure $L$ of locally bounded variation is called a resolvent of the first kind of the kernel $K \in L_{\text {loc }}^{1}\left(\mathbb{R}_{+}, \mathbb{R}\right)$ if

$$
\int_{[0, t]} K(t-s) L(d s)=1, \quad t \geq 0 .
$$

If such $L$ exists, then it is unique by Gripenberg et al. (1990, Theorem 5.2.2). We consider the following condition on the kernel $K$ :

the kernel is nonnegative, non-increasing and continuously differentiable on $(0, \infty)$, and its resolvent of the first kind $L$ is nonnegative and non-increasing in the sense that $s \mapsto L([s, s+t])$ is non-increasing for all $t \geq 0$.

We note in (2.10) that any nonnegative and non-increasing kernel that is not identically zero admits a resolvent of the first kind; see Gripenberg et al. (1990, Theorem 5.5.5). The following example provides a large class of kernels for which (2.10) is satisfied.

Example 2.4. If $K$ is completely monotone on $(0, \infty)$, then $(2.10)$ holds due to Gripenberg et al. (1990, Theorem 5.5.4). Recall that a function $f$ is called completely monotone on $(0, \infty)$ if it is infinitely differentiable there with $(-1)^{k} f^{(k)}(t) \geq 0$ for all $t>0$ and $k \geq 0$. We also note that, for each $h>0$, the shifted kernel $\Delta_{h} K$ are again completely monotone on $[0, \infty)$ so that $(2.10)$ holds also for $\Delta_{h} K$. In particular, $\Delta_{h} K \in L_{\text {loc }}^{2}\left(\mathbb{R}_{+}, \mathbb{R}\right)$, for each $h>0$. This covers, for instance, any constant positive kernel, the fractional kernel $t^{H-1 / 2}$ with $H \in(-1 / 2,1 / 2]$, and the exponentially decaying kernel $\mathrm{e}^{-\eta t}$ with $\eta>0$. Moreover, sums and products of completely monotone kernels are completely monotone. By combining the above examples we find that the Gamma kernel $K(t)=t^{H-1 / 2} \mathrm{e}^{-\eta t}$ for $H \in(-1 / 2,1 / 2]$ and $\eta \geq 0$ satisfies (2.10).

The following theorem establishes the existence of solutions to the Riccati-Volterra equation (2.4)-(2.5) under structural assumptions on $f_{0}, f_{1}, f_{2}$.

Theorem 2.5. Fix $b \in \mathbb{R}, c \geq 0$ and $\nu$ a nonnegative measure supported on $\mathbb{R}_{+}$such that $\int_{\mathbb{R}_{+}} \zeta^{2} \nu(d \zeta)<\infty$. Let $f_{0}, f_{1}, f_{2}: \mathbb{R}_{+} \mapsto \mathbb{C}$ be three continuous functions such that

$$
\Re\left(f_{0}\right)+\frac{c}{2} \Re\left(f_{1}\right)^{2}+\frac{1}{2} \int_{\mathbb{R}+} \zeta^{2} \nu(d \zeta) \Re\left(f_{2}\right)^{2} \leq 0 \quad \text { and } \quad \Re\left(f_{2}\right) \leq 0 .
$$

Fix a kernel $K \in L_{\text {loc }}^{1}\left(\mathbb{R}_{+}, \mathbb{R}\right)$ satisfying (2.10). Then, there exists a continuous solution $\psi$ to (2.4)-(2.5) such that $\Re(\psi(t)) \leq 0$, for all $t \geq 0$.

Proof. We refer to Section 5.2.

Remark 2.6. - Under (2.11), (2.8) follows from the fact that $\Re(\psi) \leq 0$ as stated in Theorem 2.5. 
- The condition (2.11) is satisfied for instance if $\Re\left(f_{0}\right) \leq 0$ and $f_{1}, f_{2}: \mathbb{R}_{+} \mapsto i \mathbb{R}$. In particular, the second part of Theorem 2.2 provides weak uniqueness for (1.1). Going back to Example 2.3, the existence of a solution to the corresponding Riccati-Volterra equation is ensured provided that $\Re\left(h_{0}\right) \leq 0$ and $h_{2}: \mathbb{R}_{+} \mapsto i \mathbb{R}$.

Remark 2.7. If $K$ is in $L_{\text {loc }}^{2}\left(\mathbb{R}_{+}, \mathbb{R}\right)$, then Theorems 2.2 and 2.5 agree with Cuchiero and Teichmann (2020, Theorem 5.12) for the jump case; if in addition $\nu \equiv 0$, then one recovers Abi Jaber et al. (2019b, Theorem 7.1) and Abi Jaber and El Euch (2019a, Theorem 2.3) for the continuous case.

\section{$2.2 \quad$ Stability and existence}

We now present our existence and stability results for solutions to (1.1). Our strategy for constructing solutions with $L^{1}$-kernels relies on an approximation argument using $L^{2}$ kernels combined with Lemma 2.1. To fix ideas, set $\nu \equiv 0$ and assume $G_{0}=\lim _{n \rightarrow \infty} G_{0}^{n}$, with $G_{0}^{n}=\int_{0}^{\cdot} g_{0}^{n}(s) d s$, for some sequence of $L_{\text {loc }}^{1}$-functions $\left(g_{0}^{n}\right)_{n \geq 1}$. Starting from a $L_{\text {loc }}^{1}$ kernel $K$, assume that there exists a sequence of $L_{\text {loc }}^{2}-$ kernels $\left(K^{n}\right)_{n \geq 1}$ such that

$$
K^{n} \rightarrow K, \quad \text { in } L_{\text {loc }}^{1}, \quad \text { as } n \rightarrow \infty .
$$

Then, for each $n \geq 1, K^{n}$ being locally square-integrable, under suitable conditions on $\left(g_{0}^{n}, K^{n}\right)$, the results in Abi Jaber et al. (2019b); Abi Jaber and El Euch (2019a) provide existence of nonnegative solution $Y^{n}$ for $(2.3)$ with $\left(g_{0}, K\right)$ replaced by $\left(g_{0}^{n}, K^{n}\right)$. Setting $X^{n}=\int_{0}^{\cdot} Y_{s}^{n} d s$, Lemma 2.1 provides a solution $X^{n}$ to $(1.1)$ for the input $\left(G_{0}^{n}, K^{n}\right)$, that is

$$
X_{t}^{n}=G_{0}^{n}(t)+\int_{0}^{t} K^{n}(t-s) Z_{s}^{n} d s,
$$

where the characteristics of $Z^{n}$ are $\left(b X^{n}, c X^{n}, 0\right)$. Provided that $\left(X^{n}\right)_{n \geq 1}$ is tight, it will admit a convergent subsequence towards a limiting process $X$. Finally, sending $n \rightarrow \infty$, one would expect $X$ to solve (1.1) for $\nu \equiv 0$.

We will adapt the same strategy in the case of jumps. Before this, we state our generic stability result.

Theorem 2.8. Assume that there exist sequences of coefficients $\left(b^{n}, c^{n}, \nu^{n}(d \zeta)\right)_{n \geq 1}$ with $\int_{\mathbb{R}} \zeta^{2} \nu^{n}(d \zeta)<\infty$, non-increasing kernels $\left(K^{n}\right)_{n \geq 1}$ and functions $\left(G_{0}^{n}\right)_{n \geq 1}$ such that

(i)

$$
b^{n} \rightarrow b, \quad c^{n}+\int_{\mathbb{R}} \zeta^{2} \nu^{n}(d \zeta) \rightarrow c+\int_{\mathbb{R}} \zeta^{2} \nu(d \zeta) \text { and } \int_{\mathbb{R}} h(\zeta) \nu^{n}(d \zeta) \rightarrow \int_{\mathbb{R}} h(\zeta) \nu(d \zeta),
$$

for any continuous and bounded function $h$ vanishing around zero, as $n \rightarrow \infty$, for some $b \in \mathbb{R}, c \geq 0$ and $\nu(d \zeta)$ a nonnegative measure such that $\int_{\mathbb{R}} \zeta^{2} \nu(d \zeta)<\infty$. 
(ii) $\int_{0}^{t}\left|K^{n}(s)-K(s)\right| d s \rightarrow 0$, as $n \rightarrow \infty, t \geq 0$, for some non-increasing kernel $K \in$ $L_{\mathrm{loc}}^{1}\left(\mathbb{R}_{+}, \mathbb{R}\right)$.

(iii) $\sup _{t \leq T}\left|G_{0}^{n}(t)-G_{0}(t)\right| \rightarrow 0$, as $n \rightarrow \infty, T \geq 0$, for some continuous function $G_{0}$.

Then, any sequence of continuous nonnegative and non-decreasing solutions $\left(X^{n}\right)_{n \geq 1}$ to (1.1) for the respective inputs $\left(G_{0}^{n}, K^{n}, b^{n}, c^{n}, \nu^{n}\right)$, is tight on the space of continuous functions $C([0, T], \mathbb{R})$ endowed with the uniform topology, for each $T>0$. Furthermore, any limit point $X$ is a continuous non-decreasing solution to (1.1) for the input $\left(G_{0}, K, b, c, \nu\right)$.

Proof. We refer to Section 4.

Remark 2.9. One notes that if $K$ satisfies (2.10), then weak uniqueness holds thanks to Theorems 2.2 and 2.5 so that one gets from Theorem 2.8 that $X^{n} \Rightarrow X$, where $X$ is the unique solution to (1.1).

The following example illustrates an application of Theorem 2.8 to the scaling limits of Hawkes processes mentioned in the introduction. We stress that the convergence of the second modified characteristic in Theorem 2.8-(i) allows to obtain continuous limiting semimartingales $Z$ from a sequence of jump semimartingales $Z^{n}$. We recall that the resolvent of the second kind $R$ of $K$ is the unique $L_{\text {loc }}^{1}$ function solution to

$$
R(t)=K(t)+\int_{0}^{t} K(t-s) R(s) d s=K(t)+\int_{0}^{t} R(t-s) K(s) d s, \quad t \geq 0 .
$$

The resolvent $R$ exists, for any kernel $K \in L_{\text {loc }}^{1}$, see Gripenberg et al. (1990, Theorems 2.3.1 and 2.3.5).

Example 2.10. Fix $n \geq 1$ and a sequence of counting processes $\left(N^{n}\right)_{n \geq 1}$ with respective intensities

$$
\lambda_{t}^{n}=g_{0}^{n}(t)+\int_{0}^{t} K^{n}(t-s) d N_{s}^{n}, \quad t \geq 0,
$$

for some continuous function $g_{0}^{n}$ and kernel $K^{n} \in L_{\text {loc }}^{1}$. Then, convolving both sides of the equation with the resolvent of the second kind $R^{n}$ of $K^{n}$ and using (2.13), leads to

$$
\lambda_{t}^{n}=g_{0}^{n}(t)+\int_{0}^{t} R^{n}(t-s) g_{0}^{n}(s) d s+\int_{0}^{t} R^{n}(t-s) d M_{s}^{n}, \quad t \geq 0,
$$

where $M^{n}=N^{n}-\int_{0}^{\cdot} \lambda_{s}^{n} d s$. Let $X^{n}$ denote the rescaled sequence of integrated accelerated intensities $X^{n}=\frac{1}{n} \int_{0}^{\dot{l}} \lambda_{n s}^{n} d s$. Then, by an application of Lemma 2.1, $X^{n}$ satisfies

$$
X_{t}^{n}=G_{0}^{n}(t)+\int_{0}^{t} \tilde{R}^{n}(t-s) Z_{s}^{n} d s
$$


with $G_{0}^{n}(t)=\int_{0}^{t}\left(\frac{1}{n} g_{0}^{n}(n s)+\int_{0}^{s} \tilde{R}^{n}(s-u) g_{0}^{n}(n u) d u\right) d s, \tilde{R}^{n}(t)=R^{n}(n t)$ and $Z_{t}^{n}=\frac{1}{n} M_{n t}^{n}$. Whence $Z^{n}$ is a pure jump martingale with jump sizes $\frac{1}{n}$ and integrated intensity $\int_{0}^{n t} \lambda_{s}^{n} d s=$ $n^{2} X_{t}^{n}$ so that its characteristics read $\left(0,0, n^{2} X^{n} \delta_{1 / n}(d \zeta)\right)$. Set $\left(b^{n}, c^{n}, \nu^{n}\right)=\left(0,0, n^{2} \delta_{1 / n}\right)$ and observe that

$$
\int_{\mathbb{R}} \zeta^{2} \nu^{n}(d \zeta)=1 \quad \text { and } \quad \int_{\mathbb{R}} h(z) \nu^{n}(d \zeta)=h(1 / n) n^{2} \rightarrow 0, \quad \text { as } n \rightarrow \infty,
$$

for any continuous and bounded function $h$ vanishing around 0 . Whence, Theorem 2.8-(i) is satisfied with $(b, c, \nu)=(0,1,0)$. Provided that $g_{0}^{n}$ and $K^{n}$ are chosen such that $\left(\tilde{R}^{n}\right)_{n \geq 1}$ and $\left(G_{0}^{n}\right)_{n \geq 1}$ satisfy Theorem 2.8-(ii)-(iii) for some $\tilde{R}$ and $G_{0}$, Theorem 2.8 yields that $\left(X^{n}\right)_{n \geq 1}$ is tight on $C([0, T], \mathbb{R})$ such that any limit point satisfies

$$
X_{t}=G_{0}(t)+\int_{0}^{t} \tilde{R}(t-s) Z_{s} d s
$$

with $Z$ a continuous martingale with characteristics $(0, X, 0)$. For instance, setting $g_{0}^{n} \equiv 0$, Jusselin and Rosenbaum (2018) construct a sequence of kernels $K^{n}$ such that $\tilde{R}^{n}$ converges in $L^{1}([0, T], \mathbb{R})$ towards the function

$$
\tilde{R}_{H}(t)=\lambda t^{\alpha-1} E_{\alpha, \alpha}\left(-\lambda t^{\alpha}\right),
$$

for some $\lambda \in \mathbb{R}, \alpha=H+1 / 2$ with $H \in(-1 / 2,1 / 2)$ and $E_{\alpha, \alpha}(z)=\sum_{n \geq 0} z^{n} / \Gamma(\alpha(n+1))$ the so-called Mittag-Leffler function. In particular, $\tilde{R}_{H}$ is the resolvent of the second kind of $\lambda K_{H}$ where $K_{H}$ is the fractional kernel given by (1.9). In this case, using again the resolvent equation (2.13), one obtains

$$
X_{t}=\int_{0}^{t} \lambda K_{H}(t-s) \tilde{Z}_{s} d s
$$

with $\tilde{Z}=X+Z$.

Remark 2.11. In the $L^{2}$ setting, i.e. when the characteristics of $Z$ are absolutely continuous with respect to the Lebesgue measure, Abi Jaber et al. (2019a, Theorem 1.6) provides a generic stability result for stochastic Volterra equations with jumps using a 'martingale problem' formulation, for general coefficients for the differential characteristics of $Z$ going beyond the affine case.

We now introduce the monotonicity and continuity assumptions needed on the kernel $K$ and the input function $G_{0}$ to construct non-decreasing and nonnegative solutions to (1.1). We assume that $K \in L_{\text {loc }}^{1}\left(\mathbb{R}_{+}, \mathbb{R}\right)$ such that (2.10) holds. Concerning the input function $G_{0}$, in the absence of jumps and for $K \in L_{\text {loc }}^{2}$ Abi Jaber and El Euch (2019a) provide a set $\mathcal{G}_{K}$ of admissible input curves $g_{0}$ defined in terms of the resolvent of the first 
kind $L$ to ensure the existence of non-negative solution for (2.3). To guarantee that the approximate solutions (2.12) are non-decreasing, we consider similarly to Abi Jaber and El Euch (2019a, Equations (2.4)-(2.5)), the following condition ${ }^{2}$

$$
\Delta_{h} g_{0}-\left(\Delta_{h} K * L\right)(0) g_{0}-d\left(\Delta_{h} K * L\right) * g_{0} \geq 0, \quad h \geq 0,
$$

where we used the notation $(f * \mu)(t)=\int_{0}^{t} f(t-s) \mu(d s)$ for a measure of locally bounded variation $\mu$ and a function $f \in L_{\text {loc }}^{1}$. We then define the space of admissible input curves $\mathcal{G}_{K}$ to be

$$
\mathcal{G}_{K}=\left\{g_{0} \text { continuous, satisfying }(2.14) \text { such that } g_{0}(0) \geq 0\right\} .
$$

Two notable examples of such admissible input curves are:

Example 2.12. (i) $g_{0}$ continuous and non-decreasing with $g_{0}(0) \geq 0$,

(ii) $g_{0}(t)=x_{0}+\int_{0}^{t} K(t-s) \theta(s) d s$, for some $x_{0} \geq 0$ and $\theta: \mathbb{R}_{+} \rightarrow \mathbb{R}_{+}$locally bounded, see e.g. Abi Jaber and El Euch (2019a, Example (2.2)).

We are now in place to state the main existence (and uniqueness) result.

Theorem 2.13. Fix $b \in \mathbb{R}, c \geq 0$ and $\nu$ a nonnegative measure supported on $\mathbb{R}_{+}$such that $\int_{\mathbb{R}_{+}} \zeta^{2} \nu(d \zeta)<\infty$. Fix a kernel $K \in L_{\mathrm{loc}}^{1}\left(\mathbb{R}_{+}, \mathbb{R}\right)$. Assume that $K$ and its shifted kernels $\Delta_{1 / n} K$ satisfy (2.10), for any $n \geq 1$. Let $G_{0}=\lim _{n \rightarrow \infty} \int_{0}^{\cdot} g_{0}^{n}(s) d s$ for some functions $g_{0}^{n} \in \mathcal{G}_{\Delta_{1 / n} K}, n \geq 1$, and assume that $G_{0}$ is continuous. Then, there exists a unique nondecreasing nonnegative continuous weak solution $X$ to (1.1) for the input $\left(G_{0}, K, b, c, \nu\right)$.

Proof. The proof for the existence part is given in Section 5.1. The uniqueness statement is obtained from Theorems 2.2 and 2.5, recall Remark 2.6.

Remark 2.14. If $K$ satisfies (2.10) and $g_{0} \in \mathcal{G}_{K}$ as in Example 2.12, the continuous function $G_{0}(t)=\int_{0}^{t} g_{0}(s) d s$ satisfies the assumption of Theorem 2.13. Indeed:

(i) Take $g_{0}$ as in Example 2.12-(i), fix $n \geq 1$ and set $g_{0}^{n}=\Delta_{1 / n} g_{0}$. Clearly, $g_{0}^{n}$ satisfies again Example 2.12-(i), so that $g_{0}^{n} \in \mathcal{G}_{\Delta_{1 / n} K}$. Furthermore, we have that $\lim _{n \rightarrow \infty} \int_{0}^{t} g_{0}^{n}(s) d s=G_{0}(t)$ by virtue of Brezis (2010, Lemma 4.3).

(ii) Take $g_{0}$ as in Example 2.12-(ii) and set $g_{0}^{n}(t)=x_{0}+\int_{0}^{t} \Delta_{1 / n} K(t-s) \theta(s) d s$, then, similarly we have that $\lim _{n \rightarrow \infty} \int_{0}^{t} g_{0}^{n}(s) d s=G_{0}(t)$ and $g_{0}^{n} \in \mathcal{G}_{\Delta_{1 / n} K}$.

\footnotetext{
${ }^{2}$ Under (2.10) one can show that $\Delta_{h} K * L$ is right-continuous and of locally bounded variation (see Abi Jaber and El Euch (2019a, Remark B.3)), thus the associated measure $d\left(\Delta_{h} K * L\right)$ is well defined.
} 


\section{A-priori estimates}

We first provide a-priori estimates for solutions to (1.1). We make use of the resolvent of the second kind $R$ of $K$ given in (2.13).

Lemma 3.1. Fix $K \in L_{\text {loc }}^{1}\left(\mathbb{R}_{+}, \mathbb{R}\right)$ and $G_{0}$ locally bounded. Assume that there exists a non-decreasing nonnegative continuous and adapted process $\tilde{X}$ satisfying

$$
\tilde{X}_{t}=G_{0}(t)+\int_{0}^{t} K(t-s) \tilde{Z}_{s} d s
$$

where $\tilde{Z}$ is a semimartingale with characteristics $\left(\tilde{B}_{t}(\omega), \tilde{C}_{t}(\omega), \tilde{\nu}_{t}(\omega, d \zeta)\right)$ such that

$$
\left|\tilde{B}_{t}(\omega)\right|+\left|\tilde{C}_{t}(\omega)\right|+\int_{[0, t] \times \mathbb{R}} \zeta^{2} \tilde{\nu}_{s}(\omega, d \zeta) \leq \kappa_{L}\left|\tilde{X}_{t}(\omega)\right|, \quad t \geq 0, \quad \text { for a.e. } \omega
$$

for some constant $\kappa_{L}$. Then, for all $T>0$,

$$
\mathbb{E}\left[\sup _{t \leq T}\left|\tilde{X}_{t}\right|^{2}\right] \leq C\left(T, \kappa_{L}\right)\left(1+\sup _{t \leq T}\left|G_{0}(t)\right|^{2}+\|K\|_{L^{1}([0, T])}^{2}\right)\left(1+\|R\|_{L^{1}([0, T])}\right),
$$

where $C\left(T, \kappa_{L}\right)>0$ depends exclusively on $\left(T, \kappa_{L}\right)$, and $R$ is the resolvent of the second kind of $C\left(T, \kappa_{L}\right)\|K\|_{L^{1}([0, T])}|K|$.

Proof. Since $\tilde{X}$ is non-decreasing, we have $\mathbb{E}\left[\sup _{t \leq T} \tilde{X}_{t}^{2}\right] \leq \mathbb{E}\left[\tilde{X}_{T}^{2}\right]$. It is therefore enough to prove the bound (3.2) for $\mathbb{E}\left[\tilde{X}_{T}^{2}\right]$. For this, fix $n \geq 1$ and define $\tau_{n}=\inf \left\{t \geq 0:\left|\tilde{X}_{t}\right| \geq\right.$ $n\} \wedge T$. Since $\tilde{X}$ is adapted with continuous sample paths, $\tau_{n}$ is a stopping time such that $\tau_{n} \rightarrow T$ almost surely as $n \rightarrow \infty$. First observe that

$$
\left|\tilde{X}_{t}\right| \mathbf{1}_{\left\{t<\tau_{n}\right\}} \leq\left|G_{0}(t)\right|+\left|\int_{0}^{t} K(t-s) \tilde{Z}_{s} \mathbf{1}_{\left\{s<\tau_{n}\right\}} d s\right| .
$$

and set $\tilde{X}_{t}^{n}=\tilde{X}_{t} \mathbf{1}_{\left\{t \leq \tau_{n}\right\}}$. An applications of Jensen's inequality on the normalized measure $|K(t)| d t /\|K\|_{L^{1}([0, T])}$ yields

$$
\begin{aligned}
\left|\tilde{X}_{t}^{n}\right|^{2} & \leq 2\left|G_{0}(t)\right|^{2}+2\left|\int_{0}^{t} K(t-s) \tilde{Z}_{s} \mathbf{1}_{\left\{s<\tau_{n}\right\}} d s\right|^{2} \\
& \leq 2 \sup _{r \leq T}\left|G_{0}(r)\right|^{2}+2\|K\|_{L^{1}([0, T])} \int_{0}^{t}|K(t-s)|\left|\tilde{Z}_{s} \mathbf{1}_{\left\{s<\tau_{n}\right\}}\right|^{2} d s .
\end{aligned}
$$

$\tilde{Z}$ admits the decomposition $\tilde{Z}=\tilde{B}+\tilde{M}^{c}+\tilde{M}^{d}$ such that $\mathbb{E}\left[\left|\tilde{M}_{t}^{c}\right|^{2}\right]=\mathbb{E}\left[\tilde{C}_{t}\right]$ and $\mathbb{E}\left[\left|\tilde{M}_{t}^{d}\right|^{2}\right]=$ $\mathbb{E}\left[\int_{[0, t] \times \mathbb{R}} \zeta^{2} \tilde{\nu}_{s}(\cdot, d \zeta)\right]$, so that Jensen's inequality combined with the bound (3.1) yield

$$
\mathbb{E}\left[\tilde{Z}_{s}^{2} \mathbf{1}_{\left\{s<\tau_{n}\right\}}\right] \leq 3\left(\kappa_{L}^{2}+\kappa_{L}\right)\left(1+\mathbb{E}\left[\left|\tilde{X}_{s}^{n}\right|^{2}\right]\right), \quad s \geq 0 .
$$


Combining the above, we get for a constant $C$ depending exclusively on $\left(T, \kappa_{L}\right)$ that may vary from line to line:

$$
\begin{aligned}
\mathbb{E}\left[\left|\tilde{X}_{t}^{n}\right|^{2}\right] & \leq C \sup _{r \leq T}\left|G_{0}(r)\right|^{2}+C\|K\|_{L^{1}([0, T])} \int_{0}^{t}|K(t-s)|\left(1+\mathbb{E}\left[\left|\tilde{X}_{s}^{n}\right|^{2}\right]\right) d s \\
& \leq C\left(1+\sup _{r \leq T}\left|G_{0}(r)\right|^{2}+\|K\|_{L^{1}([0, T])}^{2}\right)\left(1+\|R\|_{L^{1}([0, T])}\right) .
\end{aligned}
$$

where the last line follows from the generalised Gronwall inequality for convolution equations with $R$ the resolvent of $C\|K\|_{L^{1}([0, T])}^{p-1}|K|$, see Gripenberg et al. (1990, Theorem 9.8.2). The claimed estimate (3.2) now follows by sending $n \rightarrow \infty$ and using Fatou's Lemma in the above.

Remark 3.2. If $Z$ is a semimartingale with characteristics (1.2), the growth condition (3.1) is clearly satisfied with $\kappa_{L}=\left(b+c+\int_{\mathbb{R}_{+}} \zeta^{2} \nu(d \zeta)\right)$, recall the assumption $\int_{\mathbb{R}_{+}} \zeta^{2} \nu(d \zeta)<$ $\infty$. Whence, any solution to (1.1) satisfies the estimate (3.2).

The following lemma establishes an estimate for the modulus of continuity of the process $\bar{X}=X-G_{0}$ defined by

$$
w_{\bar{X}, T}(\delta)=\sup \left\{\left|\bar{X}_{s}-\bar{X}_{t}\right|: s, t \leq T \text { and }|s-t| \leq \delta\right\}, \quad 0<\delta \leq 1 .
$$

Lemma 3.3. Fix $K \in L_{\text {loc }}^{1}\left(\mathbb{R}_{+}, \mathbb{R}\right)$ non-increasing and $G_{0}$ a locally bounded function. Let $X$ denote a solution to (1.1) for the input $\left(G_{0}, K, b, c, \nu\right)$ and set $\bar{X}=X-G_{0}$. Then, for any $T>0$ and $\delta \leq 1$,

$$
\mathbb{E}\left[w_{\bar{X}, T}(\delta)\right] \leq 3\left(\kappa_{L}^{2}+\kappa_{L}\right)\left(1+\mathbb{E}\left[X_{T}^{2}\right]\right)\left(\int_{0}^{\delta}|K(s)| d s+\int_{0}^{T}(K(s)-K(s+\delta)) d s\right)
$$

with $\kappa_{L}=\left(b+c+\int_{\mathbb{R}_{+}} \zeta^{2} \nu(d \zeta)\right)$.

Proof. Fix $s, t \leq T$ such that $|t-s| \leq \delta$. We first write

$$
\bar{X}_{s}-\bar{X}_{t}=\int_{s \wedge t}^{s \vee t} K(s \vee t-u) Z_{u} d u+\int_{0}^{s \wedge t}(K(s \vee t-u)-K(s \wedge t-u)) Z_{u} d u .
$$

Whence,

$$
\begin{aligned}
\left|\bar{X}_{s}-\bar{X}_{t}\right| & \leq \sup _{u \leq T}\left|Z_{u}\right|\left(\int_{s \wedge t}^{s \vee t}|K(s \vee t-u)| d u+\int_{0}^{s \wedge t}|K(s \vee t-u)-K(s \wedge t-u)| d u\right) \\
& \leq \sup _{u \leq T}\left|Z_{u}\right|\left(\int_{0}^{\delta}|K(u)| d u+\int_{0}^{s \wedge t}|K(s \vee t-s \wedge t+u)-K(u)| d u\right) \\
& \leq \sup _{u \leq T}\left|Z_{u}\right|\left(\int_{0}^{\delta}|K(u)| d u+\int_{0}^{T}(K(u)-K(u+\delta)) d u\right)
\end{aligned}
$$


where the last inequality follows from the fact that $K$ is non-increasing and $(s \vee t-$ $s \wedge t) \leq \delta$. The claimed estimate follows upon taking the supremum over $s, t$ and the expectation, using the Burkholder-Davis-Gundy inequality for the local martingale parts of $Z$ and Remark 3.2.

\section{Tightness and stability}

In this section, we prove our general tightness and stability result: Theorem 2.8. One can appreciate the formulation (1.1) and the affine structure of the characteristics (1.2) for the stability argument.

We start with a preliminary lemma.

Lemma 4.1. Let $\left(K^{n}\right)_{n \geq 1}$ be a sequence of locally integrable kernels $K^{n}$ such that $\int_{0}^{t} \mid K^{n}(s)-$ $K(s) \mid d s \rightarrow 0$, as $n \rightarrow \infty, t \geq 0$, for some kernel $K \in L_{\text {loc }}^{1}\left(\mathbb{R}_{+}, \mathbb{R}\right)$. Then, for all $T>0$,

$$
\lim _{\delta \rightarrow 0} \limsup _{n \rightarrow \infty}\left(\int_{0}^{\delta}\left|K^{n}(s)\right| d s+\int_{0}^{T}\left|K^{n}(s+\delta)-K^{n}(s)\right| d s\right)=0 .
$$

Proof. Fix $\varepsilon>0$ and $T>0$. Since $K \in L_{\text {loc }}^{1}\left(\mathbb{R}_{+}, \mathbb{R}\right)$, it is $L^{1}$-continuous, see Brezis (2010, Lemma 4.3), so that we can fix $\delta<1$ such that

$$
\int_{0}^{\delta}|K(s)| d s+\int_{0}^{T}|K(s+\delta)-K(s)| d s \leq \frac{\varepsilon}{5} .
$$

Due to the $L^{1}$-convergence of the kernels $\left(K^{n}\right)_{n \geq 1}$, let $n_{\delta}$ be such that

$$
\int_{0}^{T+1}\left|K^{n}(s)-K(s)\right| d s \leq \frac{\varepsilon}{5}, \quad n \geq n_{\delta} .
$$

Fixing $n \geq n_{\delta}$ and using the above leads to

$$
\int_{0}^{\delta}\left|K^{n}(s)\right| d s \leq \int_{0}^{T+1}\left|K^{n}(s)-K(s)\right| d s+\int_{0}^{\delta}|K(s)| d s \leq \frac{2}{5} \varepsilon
$$

and

$$
\begin{aligned}
\int_{0}^{T}\left|K^{n}(s+\delta)-K^{n}(s)\right| d s \leq & 2 \int_{0}^{T+1}\left|K^{n}(s)-K(s)\right| d s \\
& +\int_{0}^{T}|K(s+\delta)-K(s)| d s \\
\leq & \frac{3}{5} \varepsilon .
\end{aligned}
$$

Whence

$$
\int_{0}^{\delta}\left|K^{n}(s)\right| d s+\int_{0}^{T}\left|K^{n}(s+\delta)-K^{n}(s)\right| d s \leq \varepsilon,
$$

which yields (4.1). 
Proof of Theorem 2.8. Let $\left(X^{n}\right)_{n \geq 1}$ be a sequence of continuous non-decreasing solutions to (1.1), for the respective inputs $\left(G_{0}^{n}, K^{n}, b^{n}, c^{n}, \nu^{n}\right)$, that is, for each $n \geq 1$,

$$
X_{t}^{n}=G_{0}^{n}(t)+\int_{0}^{t} K^{n}(t-s) Z_{s}^{n} d s, \quad t \geq 0,
$$

where $Z^{n}$ is a semimartingale with characteristics $\left(b^{n} X^{n}, c^{n} X^{n}, \nu^{n}(d \zeta) X^{n}\right)$, defined on some filtered probability space $\left(\Omega^{n}, \mathcal{F}^{n},\left(\mathcal{F}_{t}^{n}\right)_{t \geq 0}, \mathbb{P}^{n}\right)$.

- Fix $T>0$. We argue tightness of $\left(X^{n}, Z^{n}\right)$ on the space $C([0, T], \mathbb{R}) \times D([0, T], \mathbb{R})$, where $D([0, T], \mathbb{R})$ is the Skorokhod space endowed with the $J_{1}$ topology. To prove tightness of $\left(X^{n}\right)_{n \geq 1}$, we start by observing that due to the uniform convergence of $\left(G_{0}^{n}\right)_{n \geq 1}$ in (iii), it suffices to obtain the tighthness of the sequence $\bar{X}^{n}=X^{n}-G_{0}^{n}$. To this end, we make use of the probabilistic counterpart of the Arzéla-Ascoli theorem given in Billingsley (1999, Theorem 7.2). By Chebyshev's inequality, it suffices to prove that

$$
\sup _{n \geq 1} \mathbb{E}\left[\sup _{t \leq T} \bar{X}_{t}^{n}\right]<\infty \quad \text { and } \quad \lim _{\delta \rightarrow 0} \limsup _{n \geq \infty} \mathbb{E}\left[w_{\bar{X}^{n}, T}(\delta)\right]=0
$$

where $w$ is the modulus of continuity defined as in (3.3). By virtue of the continuous dependence of the resolvent on the kernel in $L^{1}$, the $L^{1}$-convergence of $K^{n}$ in (ii) implies the $L^{1}$-convergence of the respective sequence of resolvents $\left(R^{n}\right)_{n \geq 1}$, see Gripenberg et al. (1990, Theorem 2.3.1). Thus, the sequences $\left(\left\|K^{n}\right\|_{L^{1}([0, T])}\right)_{n \geq 1}$ and $\left(\left\|R^{n}\right\|_{L^{1}([0, T])}\right)_{n \geq 1}$ are uniformly bounded in $n$. Furthermore, it follows from (i) that $\left(b^{n}, c^{n}, \int_{\mathbb{R}} \zeta^{2} \nu^{n}(d \bar{\zeta})\right)_{n \geq 1}$ are uniformly bounded in $n$ so that the coefficient $\kappa_{L}$ appearing in (3.1) for $X^{n}$, recall Remark 3.2, does not depend on $n$. Therefore, recalling (iii), $\sup _{t \leq T} G_{0}^{n}(t)$ is uniformly bounded in $n$ and the bound in (3.2) for each $X^{n}$ does not depend on $n$, yielding $\sup _{n>1} \mathbb{E}\left[\sup _{t<T}\left|\bar{X}_{t}^{n}\right|^{2}\right]<\infty$. From there, an application of Lemmas 3.3 and 4.1 lead to (4.3) and the tightness of $\left(X^{n}\right)_{n \geq 1}$ on $C([0, T], \mathbb{R})$ follows. We claim that the sequence $\left(Z^{n}\right)_{n \in \mathbb{N}}$ is tight on $D([0, T], \mathbb{R})$. To prove this we verify the conditions in Jacod and Shiryaev (2003, Theorem VI.4.18). We first note that for any $a>0, \varepsilon>0, N \leq T$, we have

$$
\mathbb{P}\left(\int_{[0, N] \times \mathbb{R}} \mathbf{1}_{|\zeta|>a} d X_{s}^{n} \nu^{n}(d \zeta)>\varepsilon\right) \leq \frac{1}{a^{2} \varepsilon} \sup _{n \geq 1} \int_{\mathbb{R}}|\zeta|^{2} \nu^{n}(d \zeta) \sup _{m \geq 1} \mathbb{E}\left[X_{T}^{m}\right]
$$

Therefore,

$$
\lim _{a \rightarrow \infty} \sup _{n \in \mathbb{N}} \mathbb{P}\left(\int_{[0, N] \times \mathbb{R}} \mathbf{1}_{|\zeta|>a} d X_{s}^{n} \nu^{n}(d \zeta)>\varepsilon\right)=0 .
$$

Furthermore, since $\left(X^{n}\right)_{n \geq 1}$ is tight on $C([0, T], \mathbb{R})$, the first two 'modified' characteristics $\left(b^{n} X^{n},\left(c^{n}+\int_{\mathbb{R}} \zeta^{2} \nu^{n}(d \zeta)\right) X^{n}\right)$ of $Z^{n}$ are $C$-tight by virtue of (i). In addition, for $p \in \mathbb{N}$ and $h_{p}(\zeta)=(p|\zeta|-1)^{+} \wedge 1, X^{n} \int_{\mathbb{R}} h_{p}(\zeta) \nu^{n}(d \zeta)$ is also $C$-tight thanks to (i). Whence, we may apply Jacod and Shiryaev (2003, Theorem VI.4.18) to get that $\left(Z^{n}\right)_{n \in \mathbb{N}}$ is tight on 
$D([0, T], \mathbb{R})$. Finally, by passing to a further subsequence, we have $\left(X^{n}, Z^{n}\right) \Rightarrow(X, Z)$ on $C([0, T], \mathbb{R}) \times D([0, T], \mathbb{R})$ for some limiting process $(X, Z)$ defined on a probability space $(\Omega, \mathcal{F}, \mathbb{P})$.

- We now move to the stability part. We start by proving that $Z$ is a semimartingale with characteristics $(b X, c X, \nu(d \zeta) X)$ with respect to the filtration $\mathbb{F}=\left(\mathcal{F}_{t}\right)_{t \leq T}$ generated by $(X, Z)$. Since $X^{n}$ and $X$ are continuous, we have $X^{n} \Rightarrow X$ on $D([0, T], \mathbb{R})$ so that, by virtue of (i), we have, by passing to a further subsequence,

$$
\left(X^{n}, Z^{n}, b^{n} X^{n},\left(c^{n}+\int_{\mathbb{R}} \zeta^{2} \nu^{n}(d \zeta)\right) X^{n}\right) \Rightarrow\left(X, Z, b X,\left(c+\int_{\mathbb{R}} \zeta^{2} \nu(d \zeta)\right) X\right)
$$

and for any continuous and bounded function $h$ vanishing around zero:

$$
\left(X^{n}, Z^{n}, \int_{\mathbb{R}_{+}} h(\zeta) \nu^{n}(d \zeta) X^{n}\right) \Rightarrow\left(X, Z, \int_{\mathbb{R}_{+}} h(\zeta) \nu(d \zeta) X\right)
$$

It follows from Jacod and Shiryaev (2003, Theorem IX-2.4 and Remark IX-2.21) that $Z$ is a semimartingale with characteristics $(b X, c X, \nu(d \zeta) X)$ with respect to the filtration $\mathbb{F}$. An application of Skorokhod's representation theorem provides the existence of a common filtered probability space $\left(\Omega, \mathcal{F},\left(\mathcal{F}_{t}\right)_{t \geq 0}, \mathbb{P}\right)$ supporting a sequence of copies $\left(X^{n}, Z^{n}\right)_{n \geq 1}$ that converges on $C([0, T] \times \mathbb{R}) \times D([0, T] \times \mathbb{R})$, almost surely, along a subsequence, towards a copy of $(X, Z)$. Keeping the same notations for these copies, we have

$$
\left\|X^{n}-X\right\|_{C([0, T], \mathbb{R})} \rightarrow 0, \quad\left\|Z^{n}-Z\right\|_{D([0, T], \mathbb{R})} \rightarrow 0, \quad \mathbb{P}-\text { a.s., } \quad \text { as } n \rightarrow \infty .
$$

Now fix $t \leq T$ and write

$$
\begin{aligned}
\int_{0}^{t} K^{n}(t-s) Z_{s}^{n} d s-\int_{0}^{t} K(t-s) Z_{s} d s= & \int_{0}^{t}\left(K^{n}(t-s)-K(t-s)\right) Z_{s}^{n} d s \\
& +\int_{0}^{t} K(t-s)\left(Z_{s}^{n}-Z_{s}\right) d s \\
& =\mathbf{I}_{n}+\mathbf{I I}_{n} .
\end{aligned}
$$

Due to the convergence of $\left(Z^{n}\right)_{n \geq 1}$ on $D([0, T], \mathbb{R}), Z_{s}^{n} \rightarrow Z_{s} \mathbb{P} \times d t$-almost everywhere and $\sup _{n \geq 1} \sup _{s \leq T}\left|Z_{s}^{n}\right|<\infty$ so that $\mathbf{I}_{n} \rightarrow 0$ as $n \rightarrow \infty$ by virtue of (ii) and $\mathbf{I I}_{n} \rightarrow 0$ by dominated convergence. This shows that $\int_{0}^{t} K^{n}(t-s) Z_{s}^{n} d s \rightarrow \int_{0}^{t} K(t-s) Z_{s} d s$. Combined with (iii), we get, after taking the limit in (4.2), that

$$
X_{t}=\lim _{n \rightarrow \infty} X_{t}^{n}=G_{0}(t)+\int_{0}^{t} K(t-s) Z_{s} d s
$$

for all $t \leq T$. Since $X, G_{0}$ and $t \mapsto \int_{0}^{t} K(t-s) Z_{s} d s$ are continuous, one can interchange the quantifiers so that the previous identity holds for all $t \leq T, \mathbb{P}$ almost surely. Finally, each $X^{n}$ being non-decreasing and nonnegative, the limit process $X$ is again non-decreasing and nonnegative, which ends the proof. 


\section{$5 \quad$ Existence for $L^{1}$-kernels}

\subsection{Existence for the stochastic Volterra equation}

In this section we prove the existence of solutions for the stochastic Volterra equation (1.1), i.e. Theorem 2.13. We proceed in two steps. We first prove the claimed existence for smooth kernels $K \in C^{1}$ and finite measures $\nu$. Second, we apply a density argument, i.e. Theorem 2.8 , to obtain the existence for $K \in L_{\text {loc }}^{1}$ with possibly infinite activity jumps. We point out that for $L^{2}$-kernels and possibly infinite activity jumps, existence was already obtained by Cuchiero and Teichmann (2020) using infinite dimensional Markovian lifts. However, the set of admissible input curves there is different than $\mathcal{G}_{K}$, recall (2.15), and the assumptions on $K$ are different. For this reason, we provide another proof in the $L^{2}$-setting by working directly on the level of the scalar stochastic Volterra equation, in the spirit of Abi Jaber and El Euch (2019a); Abi Jaber et al. (2019b).

To this end, for a stopping time $\tau$ we extend the definition of the set $\mathcal{G}_{K}$ in (5.1) by considering

$$
\begin{array}{r}
\mathcal{G}_{K}^{\tau}=\left\{(g(s))_{s \geq 0} \text { adapted process: satisfying }(2.14)\right. \\
\text { and continuous on }[\tau(\omega), \infty) \text { with } g(\tau(\omega)) \geq 0 \text { a.s. }\} .
\end{array}
$$

The following lemma provides some elementary results on $\mathcal{G}_{K}$.

Lemma 5.1. Let $K$ be nonnegative, non-increasing and continuous on $[0, \infty)$ and $\tau$ a stopping time.

(i) Let $\eta$ be a nonnegative random variable, then $s \mapsto \mathbf{1}_{\tau \leq s} K(s-\tau) \eta$ belongs to $\mathcal{G}_{K}^{\tau}$,

(ii) If $g_{1}, g_{2} \in \mathcal{G}_{K}^{\tau}$, then $g_{1}+g_{2} \in \mathcal{G}_{K}^{\tau}$. If $\tau \leq \tau^{\prime}$, then $\mathcal{G}_{K}^{\tau} \subset \mathcal{G}_{K}^{\tau^{\prime}}$.

Proof. (ii) is straightforward from the affine structure of (2.14). We prove (i). Clearly $g: s \mapsto \mathbf{1}_{\tau \leq s} K(s-\tau) \eta$ is continuous on $\{\tau(\omega) \leq t\}$ such that $g(\tau)=K(0) \eta \geq 0$ a.s. To argue (2.14), we fix $h \geq 0$. It follows from Abi Jaber and El Euch (2019a, Lemma B.2 and Remark B.3) that

$$
\Delta_{h} K=\left(\Delta_{h} K * L\right)(0) K+d\left(\Delta_{h} K * L\right) * K
$$

Whence, on $\{\tau \leq t\}$ :

$$
\begin{aligned}
\Delta_{h} g(t) & =\Delta_{h} K(t-\tau) \eta \\
& =\left(\Delta_{h} K * L\right)(0) K(t-\tau) \eta+\left(d\left(\Delta_{h} K * L\right) * K\right)(t-\tau) \eta \\
& =\left(\Delta_{h} K * L\right)(0) g(t)+\int_{0}^{t} d\left(\Delta_{h} K * L\right)(d s) \mathbf{1}_{\tau \leq t-s} K(t-s-\tau) \eta \\
& =\left(\Delta_{h} K * L\right)(0) g(t)+\left(d\left(\Delta_{h} K * L\right) * g\right)(t)
\end{aligned}
$$

which yields that the left hand side of (2.14) is zero, leading to (i). 
We recast the existence results of Abi Jaber et al. (2019b); Abi Jaber and El Euch (2019a) obtained for $L^{2}$-kernels and deterministic input curves $g_{0} \in \mathcal{G}_{K}$ in the absence of jumps in our framework to allow for random input curves.

Lemma 5.2. Fix $\beta, \sigma \in \mathbb{R}$ and let $K \in C^{1}[0, \infty)$ satisfying $(2.10)$. Let $\left(\Omega, \mathcal{F},\left(\mathcal{F}_{t}\right)_{t \leq T}, \mathbb{P}\right)$ denote a probability space supporting a Brownian motion $W$. Fix a stopping time $\tau$ and a process $\tilde{g}_{\tau} \in \mathcal{G}_{K}^{\tau}$. Then, the equation

$$
Y_{t}=\tilde{g}_{\tau}(t)+\int_{\tau}^{t} K(t-s) \beta Y_{s} d s+\int_{\tau}^{t} K(t-s) \sigma \sqrt{Y_{s}} d W_{s}
$$

admits a unique nonnegative continuous and adapted strong solution $Y$ on $[\tau, \infty)$. Furthermore, $\mathcal{G}_{K}^{\tau}$ is invariant for the process

$$
\tilde{g}_{t}(s)=\tilde{g}_{\tau}(s)+\int_{\tau}^{t} K(s-u) \beta Y_{u} d s+\int_{\tau}^{t} K(s-u) \sigma \sqrt{Y_{u}} d W_{u}, \quad \tau \leq t \leq s,
$$

meaning that $g_{t}$ is $\mathcal{G}_{K}^{\tau}$-valued on $\left.\left.\llbracket \tau, \infty\right)\right)=\{(\omega, t): \tau(\omega) \leq t\}$.

Proof. We first argue for $\tau \equiv 0$ and deterministic input $g_{0} \in \mathcal{G}_{K}$. The existence of a $\mathbb{R}_{+}$-valued continuous nonnegative weak solution $Y$ to (5.2) follows from Abi Jaber and El Euch (2019a, Theorem A.2) ${ }^{3}$. The strong uniqueness of $Y$ follows from Abi Jaber and El Euch (2019b, Proposition B.3). This yields the strong existence and uniqueness for (5.2). Finally, an application of the second part of Abi Jaber and El Euch (2019a, Theorem 3.1) yields the invariance of $\mathcal{G}_{K}$ with respect to $t \mapsto \tilde{g}_{t}$, after noticing that

$$
\tilde{g}_{t}(s)=\mathbb{E}\left[Y_{s}-\int_{t}^{s} K(s-u) \beta Y_{u} d u \mid \mathcal{F}_{t}\right], \quad t \leq s .
$$

For arbitrary $\tau$ and random input $\tilde{g}_{\tau} \in \mathcal{G}_{K}^{\tau}$, the result follows by a straightforward adaptation of the aforementioned results.

We now construct a solution to (1.1) when $\nu$ is finite and $K$ is continuously differentiable by pasting continuous solutions $Y^{i}$ to $(5.2)$ on each interval $\left[\tau_{i}, \tau_{i+1}\right)$ between two consecutive jumps.

Lemma 5.3. Let $b \in \mathbb{R}, c \geq 0$ and $\nu$ be a nonnegative finite measure supported on $\mathbb{R}_{+}$such that $\int_{\mathbb{R}_{+}} \zeta^{2} \nu(d \zeta)<\infty$. Fix $K \in C^{1}[0, \infty)$ satisfying (2.10) and let $g_{0} \in \mathcal{G}_{K}$. There exists a non-decreasing nonnegative continuous solution $X$ to (1.1) for the input $G_{0}(t)=\int_{0}^{t} g_{0}(s) d s$.

\footnotetext{
${ }^{3}$ We note that all the assumptions are met there, except for the local Hölder continuity of $g_{0}$. This assumption is only needed to get Hölder sample paths of $X$, which we do not require here. Assumption $\left(H_{0}\right)$ there is satisfied with $\gamma=2$ since $K$ is $C^{1}$.
} 
Proof. Using Lemma 2.1, it is enough to first prove the existence of a càdlàg nonnegative solution $Y$ to the equation

$$
Y_{t}=g_{0}(t)+\int_{0}^{t} K(t-s) d Z_{s}
$$

where $Z$ is a semimartingale with differential characteristics with respect to the Lebesgue measure $(b Y, c Y, \nu(d \zeta) Y)$, and then set $X=\int_{0}^{\cdot} Y_{s} d s$ to obtain the desired solution to (1.1). Since $\nu$ is finite, finding a solution to equation (5.3) is equivalent to solving

$$
\begin{aligned}
Y_{t}=g_{0}(t)+\sum_{i \geq 0} \mathbf{1}_{\tau_{i} \leq t} \int_{\tau_{i}}^{t \wedge \tau_{i+1}} K(t-s)\left(\beta Y_{s} d s+\sqrt{c Y_{s}} d W_{s}\right) \\
+\sum_{i \geq 1} \mathbf{1}_{\tau_{i} \leq t} K\left(t-\tau_{i}\right) J_{i}
\end{aligned}
$$

where $\beta=b-\int_{\mathbb{R}_{+}} \zeta \nu(d \zeta), J_{i}$ are the jump sizes of $Z$ distributed according to $\nu(d \zeta) / \nu\left(\mathbb{R}_{+}\right)$ and arriving at the jump times $\tau_{i}$ with instantaneous intensity $Y_{t} \nu\left(\mathbb{R}_{+}\right)$and $W$ is a Brownian motion on some filtered probability space $\left(\Omega, \mathcal{F},\left(\mathcal{F}_{t}\right)_{t \geq 0}, \mathbb{P}\right)$.

Our strategy for constructing a solution $Y$ to the above equation consists in pasting continuous solutions $Y^{i}$ on each interval $\left[\tau_{i}, \tau_{i+1}\right)$ between two consecutive jumps. More precisely, fix a filtered probability space $\left(\Omega, \mathcal{F},\left(\mathcal{F}_{t}\right)_{t \geq 0}, \mathbb{P}\right)$ supporting a Brownian motion $W$ and a sequence of independent random variables $\left(E_{i}, U_{i}\right)_{i \geq 1}$ with $E_{i}$ exponentially distributed with intensity 1 and $U_{i}$ standard uniform, and let $F_{\nu}$ denote the cumulative distribution function with density $\nu(d \zeta) / \nu\left(\mathbb{R}_{+}\right)$. We set $\tau_{0}=0$ and we assume that for each $i \geq 0$ we have a unique nonnegative continuous solution $Y^{i}$ on $\left[\tau_{i}, \tau_{i+1}\right)$ for the following system of inductive equations

$$
\begin{aligned}
Y_{t}^{i} & =\tilde{g}^{i}(t)+\int_{\tau_{i}}^{t} K(t-s)\left(\beta Y_{s}^{i} d s+\sqrt{c Y_{s}^{i}} d W_{s}\right) \\
\tilde{g}^{i}(t) & :=g_{0}(t)+\sum_{j=0}^{i-1} \int_{\tau_{j}}^{\tau_{j+1}} K(t-s)\left(\beta Y_{s}^{j} d s+\sqrt{c Y_{s}^{j}} d W_{s}\right)+\sum_{j=1}^{i} K\left(t-\tau_{j}\right) J_{j}
\end{aligned}
$$

with the convention that $\sum_{i=0}^{-1}=\sum_{j=1}^{0}=0, \tau_{i+1}=\tau_{i}+\delta_{i}$ with $\delta_{i}=\inf \{s>0$ : $\nu\left(\mathbb{R}_{+}\right) \int_{\tau_{i}}^{\tau_{i}+s} Y_{u}^{i} d u \geq E_{i}$ \} (with the convention that $\inf \emptyset=\infty$ ) and $J_{i}=F_{\mu}^{-}\left(U_{i}\right)$. Then, by a localization argument $\tau_{i} \rightarrow \infty$ and it is straightforward to check that the process $Y$ defined by

$$
Y_{t}=\sum_{i \geq 0} Y_{t}^{i} \mathbf{1}_{\tau_{i} \leq t<\tau_{i+1}}
$$

is the unique càdlàg continuous nonnegative solution to (5.4). By the first part of Lemma 5.2, the existence and uniqueness of a solution $Y^{i}$ is ensured provided that the process $\tilde{g}^{i}$ is 
$\mathcal{G}_{K}^{\tau_{i}}$-valued, for each $i \geq 1$. We now prove this claim by induction using the second part of Lemma 5.2. Initialization: for $i=0, \tilde{g}^{0}=g_{0}$ is deterministic and $\mathcal{G}_{K}$-valued by assumption. Heredity: fix $i \geq 0$ and assume that $\tilde{g}^{i} \in \mathcal{G}_{K}^{\tau_{i}}$-valued. Fix $Y^{i}$ the unique nonnegative solution to $(5.5)$ on $\left[\tau_{i}, \infty\right)$ obtained from Lemma 5.2 for the input $\tilde{g}^{i}$. The second part of Lemma 5.2 yields that the process

$$
\tilde{g}_{t}^{i}=\tilde{g}^{i}+\int_{\tau_{i}}^{t} K(\cdot-u) \beta Y_{u}^{i} d s+\int_{\tau_{i}}^{t} K(\cdot-u) \sqrt{c Y_{u}^{i}} d W_{u}
$$

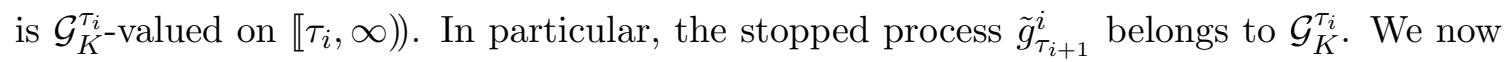
observe that (5.6) can be re-written in terms of $\tilde{g}_{\tau_{i+1}}^{i}$ :

$$
\begin{aligned}
\tilde{g}^{i+1}(t) & =\tilde{g}^{i}(t)+\int_{\tau_{i}}^{\tau_{i+1}} K(t-s)\left(\beta Y_{s}^{i} d s+\sqrt{c Y_{s}^{i}} d W_{s}\right)+K\left(t-\tau_{i+1}\right) J_{i+1} \\
& =\tilde{g}_{\tau_{i+1}}^{i}(t)+K\left(t-\tau_{i+1}\right) J_{i+1},
\end{aligned}
$$

on $\left\{t \geq \tau_{i+1}\right\}$. Since $K$ is nonnegative and $J_{i+1} \geq 0$, recall that $\nu$ is supported on $\mathbb{R}_{+}$, an application of Lemma 5.1 yields that $\tilde{g}^{i+1} \in \mathcal{G}_{K}^{\tau_{i+1}}$. This proves the induction and ends the proof.

For the general case, we use a density argument, i.e. Theorem 2.8 , to obtain the existence statement in Theorem 2.13.

Proof of the existence statement in Theorem 2.13. Fix $n \geq 1$. Let $\nu^{n}(d \zeta)=\mathbf{1}_{\zeta \geq \frac{1}{n}} \nu(d \zeta)$ and $K^{n}=\Delta_{1 / n} K$. Then, $\nu^{n}$ is a nonnegative finite measure supported on $\mathbb{R}_{+}^{n}$ such that $\int_{\mathbb{R}_{+}} \zeta^{2} \nu^{n}(d \zeta)<\infty$. An application of Lemma 5.3 yields the existence of a nondecreasing and continuous process $X^{n}$ solution to (1.1) with the inputs $\left(G_{0}^{n}, K^{n}, b, c, \nu^{n}\right)$, where $G_{0}^{n}=\int_{0}^{\cdot} g_{0}^{n}(s) d s$. Each $X^{n}$ being non-decreasing, the claimed existence now follows from Theorem 2.8, once we prove that conditions (i)-(iii) are satisfied. (i) is clearly satisfied. (ii) holds by the $L^{1}$-continuity of the kernel $K$, see Brezis (2010, Lemma 4.3). Finally, to obtain (iii) we first observe that $g_{0}^{n}$ is nonnegative, this follows from (2.14) evaluated at $t=0$. Whence, $G_{0}^{n}$ is non-decreasing with a continuous pointwise limit $G_{0}$. An application of Dini's second theorem yields (iii). The proof is complete.

\subsection{Existence for the Riccati-Volterra equation}

In this section we prove Theorem 2.5.

Proof of Theorem 2.5. We first note that the function $z \mapsto \int_{\mathbb{R}_{+}}\left(e^{z \zeta}-1-z \zeta\right) \nu(d \zeta)$ is continuous on $\mathcal{U}=\{z \in \mathbb{C}: \Re(z) \leq 0\}$. Whence, since $f_{2}$ is continuous and $\Re\left(f_{2}\right) \leq 0$ by (2.11), we obtain that $(s, u) \mapsto \int_{\mathbb{R}_{+}}\left(e^{\left(f_{2}(s)+u\right) \zeta}-1-\left(f_{2}(s)+u\right) \zeta\right) \nu(d \zeta)$ is continuous on $[0, T] \times \mathcal{U}$. We define

$$
\tilde{F}(s, u)=F\left(s, \Re(u) \mathbf{1}_{\Re(u) \leq 0}+i \Im(u)\right),
$$


where we recall that $F$ is given by (2.5). Then, $\tilde{F}$ is continuous on $[0, T] \times \mathbb{C}$ so that an application of Gripenberg et al. (1990, Theorem 12.1.1 and the comment below) (on the positive and negative parts) yields the local existence of a continuous solution $\psi$ to the equation

$$
\psi(t)=\int_{0}^{t} K(t-s) \tilde{F}(s, \psi(s)) d s
$$

on the interval $\left[0, T_{\infty}\right)$ where $T_{\infty}=\inf \{t:|\psi(t)|=\infty\}$. In order to obtain the claimed existence for (2.4)-(2.5) it suffices to prove that $T_{\infty}=+\infty$ and that $\Re(\psi(t)) \leq 0$, for all $t \geq 0$.

Step 1. We first prove that $\Re(\psi) \leq 0$ on $\left[0, T_{\infty}\right)$. Fix $T<T_{\infty}$ and denote by $\tilde{\psi}_{\mathrm{r}}(t)=$ $\Re(\psi(t)) \mathbf{1}_{\Re(\psi(t)) \leq 0}$. Taking real parts in (5.7), we get that $\chi=\Re(\psi)$ satisfies

$$
\chi(t)=\int_{0}^{t} K(t-s) \Re(\tilde{F}(s, \psi(s)) d s, \quad t \leq T,
$$

with

$$
\begin{aligned}
\Re(\tilde{F}(s, \psi(s))= & \Re\left(f_{0}(s)\right)+\frac{1}{2} c \Re\left(f_{1}(s)\right)^{2}-\frac{c}{2}\left(\Im\left(f_{1}(s)\right)+\Im(\psi(s))\right)^{2} \\
& +\left(b+c \Re\left(f_{1}(s)\right)+\frac{c}{2} \tilde{\psi}_{\mathrm{r}}(s)\right) \tilde{\psi}_{\mathrm{r}}(s) \\
& +\int_{\mathbb{R}_{+}}\left(\cos \left(\Im\left(f_{2}(s)+\psi(s)\right) \zeta\right) e^{\left(\Re\left(f_{2}(s)\right)+\tilde{\psi}_{\mathrm{r}}(s)\right) \zeta}-1-\left(\Re\left(f_{2}(s)\right)+\tilde{\psi}_{\mathrm{r}}(s)\right) \zeta\right) \nu(d \zeta) .
\end{aligned}
$$

Since $\cos \leq 1$ we obtain, for $v \leq 0$,

$$
\cos (a) e^{v \zeta}-1-v \zeta \leq e^{v \zeta}-1-v \zeta=\zeta^{2} \int_{v}^{0} \int_{r}^{0} e^{v l} d l d r \leq \frac{\zeta^{2} v^{2}}{2}
$$

Whence,

$$
\Re\left(\tilde{F}(s, \psi(s)) \leq z(s) \tilde{\psi}_{\mathrm{r}}(s)+w(s), \quad s \leq T,\right.
$$

with

$$
\begin{aligned}
& z(s)=b+c \Re\left(f_{1}(s)\right)+\frac{c}{2} \tilde{\psi}_{\mathrm{r}}(s)+\int_{\mathbb{R}_{+}} \zeta^{2} \nu(d \zeta)\left(\Re\left(f_{2}(s)\right)+\frac{1}{2} \tilde{\psi}_{\mathrm{r}}(s)\right) \\
& w(s)=\Re\left(f_{0}(s)\right)+\frac{1}{2} c \Re\left(f_{1}(s)\right)^{2}+\frac{1}{2} \int_{\mathbb{R}_{+}} \zeta^{2} \nu(d \zeta)\left(\Re\left(f_{2}(s)\right)^{2}-\frac{c}{2}\left(\Im\left(f_{1}(s)\right)+\Im(\psi(s))\right)^{2} .\right.
\end{aligned}
$$

Observing that $\tilde{\psi}_{\mathrm{r}}(t)=\mathbf{1}_{\Re(\psi(t)) \leq 0} \chi(t)$, recalling that $K$ is nonnegative and combining (5.8)-(5.9) leads to

$$
\chi(t) \leq \int_{0}^{t} K(t-s)(\tilde{z}(s) \chi(s)+w(s)) d s,
$$


with $\tilde{z}(s)=\mathbf{1}_{\Re(\psi(s)) \leq 0} z(s)$. Whence,

$$
\chi(t)=-h(t)+\int_{0}^{t} K(t-s)(\tilde{z}(s) \chi(s)+w(s)) d s
$$

for some nonnegative function $h$. Denoting by $\tilde{\chi}$ the solution to the linear equation

$$
\tilde{\chi}(t)=\int_{0}^{t} K(t-s)(\tilde{z}(s) \tilde{\chi}(s)+w(s)) d s
$$

we obtain that $\delta=(\tilde{\chi}-\chi)$ solves the linear equation

$$
\delta(t)=h(t)+\int_{0}^{t} K(t-s) \tilde{z}(s) \delta(s) d s .
$$

Since, $h \geq 0$, an application of Abi Jaber and El Euch (2019b, Theorem C.1) ${ }^{4}$ leads to $\delta \geq 0$ on $[0, T]$ so that

$$
\chi(t) \leq \tilde{\chi}(t), \quad t \leq T .
$$

We now argue that $\tilde{\chi} \leq 0$. By virtue of $(2.11), w(s) \leq 0$ for all $s \leq T$, so that another application of Abi Jaber and El Euch (2019b, Theorem C.1) on the equation (5.10) leads to $\tilde{\chi}(t) \leq 0$, for all $t \leq T$. Finally, from (5.11), we obtain that $\Re(\psi(t))=\chi(t) \leq \tilde{\chi}(t) \leq 0$, for all $t \leq T$. The claimed conclusion follows by arbitrariness of $T<T_{\infty}$.

Step 2. We now argue that $T_{\infty}=\infty$. Fix $T<T_{\infty}$. By the above we have $\tilde{\psi}_{\mathrm{r}}=\Re(\psi)$ so that $\tilde{F}(s, \psi(s))=F(s, \psi(s))$ for all $s<T_{\infty}$. Using this fact in (5.7), we observe that $\psi$ solves the linear equation

$$
h(t)=\int_{0}^{t} K(t-s)\left(\frac{c \psi(s)}{2} h(s)+\alpha(s)\right) d s
$$

with $\alpha$ defined by

$$
\alpha(s)=F(s, \psi(s))-\frac{c}{2} \psi(s)^{2} .
$$

Since $\Re(\psi) \leq 0$, an application of Abi Jaber and El Euch (2019b, Theorem C.4) ${ }^{5}$ yields that $\sup _{t \leq T}|\psi(t)|<\infty$. By arbitrariness of $T$ we obtain that $T_{\infty}=\infty$. The proof is complete.

\footnotetext{
${ }^{4}$ Inspecting the proof of Abi Jaber and El Euch (2019b, Theorem C.1) one can see that the $L^{2}$ integrability on the kernel assumed there does not play any role, the result remains clearly valid for $K \in L^{1}([0, T], \mathbb{R})$. Similarly, the continuity assumption on $z$ there can be weakened to local boundedness.

${ }^{5}$ Again the $L^{2}$ integrability on the kernel assumed there does not play any role, the result remains clearly valid for $K \in L^{1}([0, T], \mathbb{R})$.
} 


\section{Weak uniqueness and the Fourier-Laplace transform}

In this section, we prove Theorem 2.2.

Throughout this section, we fix $T \geq 0, f_{0}, f_{1}, f_{2}:[0, T] \rightarrow \mathbb{C}$ continuous functions, $G_{0}$ a non-decreasing continuous function and $K \in L^{1}([0, T], \mathbb{R})$. We let $\psi \in C([0, T], \mathbb{C})$ denote a solution to the Riccati equation (2.4)-(2.5) such that (2.8) holds and $X$ be a non-decreasing nonnegative continuous weak solution to (1.1) for the input $\left(G_{0}, K, b, c, \nu\right)$. We recall the decomposition of $Z$ in (2.1) and we define the process $V^{T}$ :

$$
\begin{aligned}
V_{t}^{T}= & V_{0}^{T}+\int_{0}^{t} \beta_{s}^{T} d X_{s}+\int_{0}^{t}\left(f_{1}(T-s)+\psi(T-s)\right) d M_{s}^{c} \\
& +\int_{0}^{t}\left(f_{2}(T-s)+\psi(T-s)\right) d M_{s}^{d} \\
\beta_{s}^{T}=- & \frac{c}{2}\left(f_{1}(T-s)+\psi(T-s)\right)^{2} \\
& \quad-\int_{\mathbb{R}_{+}}\left(e^{\left(f_{2}(T-s)+\psi(T-s)\right) \zeta}-1-\left(f_{2}(T-s)+\psi(T-s)\right) \zeta\right) \nu(d \zeta) \\
V_{0}^{T}= & \int_{0}^{T} F(T-s, \psi(T-s)) d G_{0}(s) .
\end{aligned}
$$

We note that the integral involving $\nu$ is well-defined by virtue of (2.8) combined with the local boundedness of $\left(\psi, f_{2}\right)$ and the inequality $\left|e^{\alpha \zeta}-1-\alpha \zeta\right| \leq e^{\Re(\alpha) \zeta} \alpha \zeta^{2} / 2$. The LebesgueStieltjes integrals are well-defined since $\left(\psi, f_{1}, f_{2}\right)$ are continuous and $\left(G_{0}, X\right)$ are of locally bounded variation.

$\left(V^{T}\right)_{t \leq T}$ is a semimartingale and a straightforward application of Itô's Lemma yields that the stochastic exponential $H=\exp \left(V^{T}\right)$ is a complex local martingale with dynamics

$d H_{t}=H_{t-} d N_{t}$

$d N_{t}=\left(f_{1}(T-t)+\psi(T-t)\right) d M_{t}^{c}+\int_{\mathbb{R}_{+}}\left(e^{\left(f_{2}(T-t)+\psi(T-t)\right) \zeta}-1\right)\left(\mu^{Z}(d t, d \zeta)-\nu(d \zeta) d X_{t}\right)$,

meaning that $H=\mathcal{E}(N)$, where $\mathcal{E}$ stands for the Doléans-Dade exponential. The following lemma, which extends Abi Jaber et al. (2019b, Lemma 7.3), establishes that $H$ is even a true martingale.

Lemma 6.1. Let $g_{1}, g_{2} \in L^{\infty}\left(\mathbb{R}_{+}, \mathbb{R}\right)$ such that

$$
\sup _{s \geq 0} \int_{\mathbb{R}_{+}} e^{g_{2}(s) \zeta} \zeta^{2} \nu(d \zeta)<\infty
$$

and define

$$
U_{t}=\int_{0}^{t} g_{1}(s) d M_{s}^{c}+\int_{[0, t] \times \mathbb{R}_{+}}\left(e^{g_{2}(s) \zeta}-1\right)\left(\mu^{Z}(d s, d \zeta)-\nu(d \zeta) d X_{s}\right) .
$$


Then, the Doléans-Dade exponential $\mathcal{E}(U)$ is a martingale. Furthermore, $H=\exp \left(V^{T}\right)$ is a martingale on $[0, T]$.

Proof. Part 1. Martingality of $M:=\mathcal{E}(U)$. We first recall that

$$
M_{t}=\mathcal{E}\left(\int_{0} g_{1}(s) d M_{s}^{c}\right) \prod_{0<s \leq t}\left(1+\Delta U_{s}\right) e^{-\Delta U_{s}} .
$$

Since $\Delta U_{s}=\left(e^{g_{2}(s) \Delta Z_{s}}-1\right)>-1$, for all $s \geq 0, M$ is a nonnegative local martingale. Whence, it is a supermartingale by Fatou's lemma, and it suffices to show that $\mathbb{E}\left[M_{T}\right]=1$ for any $T \in \mathbb{R}_{+}$. To this end, fix $T>0$ and define the stopping times $\tau_{n}=\inf \left\{t \geq 0: X_{t}>\right.$ $n\} \wedge T$. We first argue that $M^{\tau_{n}}=M_{\tau_{n} \wedge}$. is a uniformly integrable martingale for each $n$ by verifying the condition in Lépingle and Mémin (1978, Theorem IV.3) with the process $y(\omega, t, \zeta)=1_{t \leq \tau_{n}(\omega)}\left(e^{g_{2}(t) \zeta}-1\right)$. Using the bound $\left|\alpha \zeta e^{\alpha \zeta}+1-e^{\alpha \zeta}\right| \leq \zeta^{2} \alpha^{2} e^{\alpha \zeta} / 2$ and the boundedness of $g_{1}, g_{2}$, we get that the quantity

$$
\int_{0}^{T} \mathbf{1}_{s \leq \tau_{n}} g_{1}(s) c d X_{s}+\int_{[0, T] \times \mathbb{R}_{+}} \mathbf{1}_{s \leq \tau_{n}}\left(g_{2}(s) \zeta e^{g_{2}(s) \zeta}+1-e^{g_{2}(s) \zeta}\right) \nu(d \zeta) d X_{s}
$$

is bounded by $\kappa n\left(1+\sup _{s \geq 0} \int_{\mathbb{R}_{+}} e^{g_{2}(s) \zeta} \zeta^{2} \nu(d \zeta)\right)$, for some constant $\kappa>0$. The upper bound is finite due to condition (6.4). Lépingle and Mémin (1978, Theorem IV.3) can be applied to get that $M^{\tau_{n}}$ is a martingale for each $n$. Whence,

$$
1=M_{0}^{\tau_{n}}=\mathbb{E}_{\mathbb{P}}\left[M_{T}^{\tau_{n}}\right]=\mathbb{E}_{\mathbb{P}}\left[M_{T} \mathbf{1}_{\tau_{n} \geq T}\right]+\mathbb{E}_{\mathbb{P}}\left[M_{\tau_{n}} \mathbf{1}_{\tau_{n}<T}\right]
$$

where we made the dependence of the expectation on $\mathbb{P}$ explicit. Since $\mathbb{E}_{\mathbb{P}}\left[M_{T} \mathbf{1}_{\tau_{n} \geq T}\right] \rightarrow$ $\mathbb{E}_{\mathbb{P}}\left[M_{T}\right]$ as $n \rightarrow \infty$, by dominated convergence, in order to get that $\mathbb{E}_{\mathbb{P}}\left[M_{T}\right]=1$, it suffices to prove that

$$
\mathbb{E}_{\mathbb{P}}\left[M_{\tau_{n}} \mathbf{1}_{\tau_{n}<T}\right] \rightarrow 0, \quad \text { as } n \rightarrow \infty
$$

To this end, since $M^{\tau_{n}}$ is a martingale, we may define probability measures $\mathbb{Q}^{n}$ by

$$
\frac{d \mathbb{Q}^{n}}{d \mathbb{P}}=M_{\tau_{n}}^{\tau_{n}}
$$

By Girsanov's theorem, see Jacod and Shiryaev (2003, Theorem III.3.24) (see also the formulation in Kallsen (2006, Proposition 2.6)), the process $Z$ is a semimartingale under $\mathbb{Q}^{n}$ with characteristics

$$
\left(B^{n}, c X, \int_{0}^{\cdot} \mathbf{1}_{\left\{s \leq \tau_{n}\right\}} e^{g_{2}(s) \zeta} \nu(d \zeta) d X_{s}\right)
$$


where

$$
B^{n}=b X+\int_{0}^{\cdot} \mathbf{1}_{\left\{s \leq \tau_{n}\right\}} g_{1}(s) c d X_{s}+\int_{\left[0, \cdot \times \mathbb{R}_{+}\right.} \zeta\left(e^{g_{2}(s) \zeta}-1\right) \mathbf{1}_{\left\{s \leq \tau_{n}\right\}} \nu(d \zeta) d X_{s} .
$$

Under $\mathbb{Q}^{n}$, we still have

$$
X_{t}=G_{0}(t)+\int_{0}^{t} K(t-s) Z_{s} d s
$$

and we observe that, due to the boundedness of $g_{1}$, the equality $\left|e^{\alpha \zeta}-1\right| \leq \zeta\left(1+e^{\alpha \zeta}\right)$ and (6.4), the characteristics of $Z$ under $\mathbb{Q}^{n}$ satisfy the growth condition in $X$ as in (3.1) for some constant $\kappa_{L}$ independent of $n$. Therefore, an application of Lemma 3.1 yields the moment bound

$$
\mathbb{E}_{\mathbb{Q}^{n}}\left[\sup _{t \leq T}\left|X_{t}\right|^{2}\right] \leq \eta\left(\kappa_{L}, T, K, G_{0}\right)
$$

where $\eta\left(\kappa_{L}, T, K, G_{0}\right)$ does not depend on $n$. We then get by an application of Chebyshev's inequality

$$
\begin{aligned}
\mathbb{E}_{\mathbb{P}}\left[M_{\tau_{n}} \mathbf{1}_{\tau_{n} \leq T}\right] & =\mathbb{Q}^{n}\left(\tau_{n}<T\right) \\
& \leq \mathbb{Q}^{n}\left(\sup _{t \leq T} X_{t}>n\right) \\
& \leq \frac{1}{n^{2}} \mathbb{E}_{\mathbb{Q}^{n}}\left[\sup _{t \leq T} X_{t}^{2}\right] \\
& \leq \frac{1}{n^{2}} \eta\left(\kappa_{L}, T, K, G_{0}\right) .
\end{aligned}
$$

Sending $n \rightarrow \infty$, we obtain (6.6), proving that $M$ is martingale.

Part 2. Martingality of $H=\exp \left(V^{T}\right)$. To show that the local martinglae $H$ is a true martingale, it is enough to bound it by a martingale, see Jarrow (2018, Lemma 1.4). We fix $t \leq T$ and define $g_{i}(s)=\Re\left(f_{i}(T-s)+\psi(T-s)\right) \mathbf{1}_{s \leq T}$, and $m_{i}(s)=\Im\left(f_{i}(T-s)+\psi(T-\right.$ $s)) \mathbf{1}_{s \leq T}$ for $i=1,2$. Taking real parts in (6.1) yield

$$
\begin{aligned}
\Re\left(V_{t}^{T}\right)= & V_{0}^{T}-\frac{c}{2} \int_{0}^{t}\left(g_{1}^{2}(s)-m_{1}^{2}(s)\right) d X_{s}+\int_{0}^{t} g_{1}(s) d M_{s}^{c} \\
& +\int_{\mathbb{R}_{+}}\left(\cos \left(m_{2}(s) \zeta\right) e^{g_{2}(s) \zeta}-1-g_{2}(s) \zeta\right) \nu(d \zeta)+\int_{0}^{t} g_{2}(s) d M_{s}^{d} .
\end{aligned}
$$

Whence, using that cos is bounded by 1 , we get

$$
\left|H_{t}\right|=\exp \left(\Re\left(V_{t}^{T}\right)\right) \leq \exp \left(V_{0}^{T}+\frac{c}{2} m_{1}^{2}(s)\right) \mathcal{E}\left(U_{t}\right)
$$

with $U$ given by (6.5). Since, $\left(\psi, f_{1}, f_{2}\right)$ are continuous and satisfy (2.8), $m_{i}, g_{i}$ are bounded for $i=1,2$, and $g_{2}$ satisfies (6.4), so that $\left|H_{t}\right| \leq c_{T} \mathcal{E}\left(U_{t}\right)$ for some constant $c_{T}$ and $\mathcal{E}(U)$ is a martingale thanks to the first part. This proves that $H$ is a martingale on $[0, T]$. 
Lemma 6.2. Assume that the shifted kernels $\Delta_{h} K$ are in $L^{2}([0, T], \mathbb{R})$, for all $h>0$. Set $\left(G_{t}\right)_{t \geq 0}$ and $\left(g_{t}\right)_{t \geq 0}$ as in (2.6)-(2.7). Then, the process $\left(V_{t}^{T}\right)_{0 \leq t \leq T}$ defined by (6.1)-(6.2)(6.3) satisfies

$$
\begin{gathered}
V_{t}^{T}=\int_{0}^{t} f_{0}(T-s) d X_{s}+\int_{0}^{t} f_{1}(T-s) d M_{s}^{c}+\int_{0}^{t} f_{2}(T-s) d M_{s}^{d} \\
+\int_{t}^{T} F(T-s, \psi(T-s)) d G_{t}(s), \quad t \leq T
\end{gathered}
$$

Proof. We fix $t \leq T, h>0$ and we define

$$
\begin{aligned}
X_{t}^{h} & =G_{0}(t)+\int_{0}^{t} \Delta_{h} K(t-s) Z_{s} d s, \\
g_{t}^{h}(s) & =\int_{0}^{t} \Delta_{h} K(s-u) d Z_{u}, \\
\psi^{h}(t) & =\int_{0}^{t} \Delta_{h} K(t-s) F(s, \psi(s)) d s,
\end{aligned}
$$

where we recall that $Z=b X+M^{c}+M^{d}$ and $F$ is given by (2.5). We stress that the right-hand sides of all three quantities are defined from $X$ and $\psi$ and do not depend on $X^{h}$ or $\psi^{h} ; X_{t}^{h}$ and $g_{t}^{h}$ are well-defined as Itô integrals since $\Delta_{h} K \in L^{2}([0, T], \mathbb{R})$.

Step 1. Convergence of $X^{h}, g^{h}, \psi^{h}$. It follows from the boundedness of $\left(\psi, f_{0}, f_{1}, f_{2}\right)$ and $Z(\omega)$, condition (2.8) and the $L^{1}$-continuity of the kernel $K$ that

$$
\sup _{s \leq T}\left|\psi^{h}(s)-\psi(s)\right| \rightarrow 0, \quad\left|X_{t}^{h}-X_{t}\right| \rightarrow 0, \quad \mathbb{P}-\text { a.s. }
$$

as $h \rightarrow 0$. Set

$$
G_{t}^{h}(s)=G_{0}(s)+\int_{t}^{s \vee t} g_{t}^{h}(u) d u .
$$

By invoking a stochastic Fubini theorem, justified by the $L^{2}$-integrability of $\Delta_{h} K$, we get, for all $s>t$,

$$
G_{t}^{h}(s)-G_{t}(s)=\int_{0}^{s}\left(\Delta_{h} K(u)-K(u)\right)\left(Z_{t \wedge(s-u)}-Z_{t-u}\right) d u .
$$

The boundedness of $Z(\omega)$ and the $L^{1}$-continuity of the kernel $K$, lead to

$$
G_{t}^{h}(s) \rightarrow G_{t}(s), \quad \mathbb{P}-a . s .
$$

as $h \rightarrow 0$, for all $s \in(t, T]$.

Step 2. Proving (6.7). An application of a stochastic Fubini theorem, see Veraar (2012, 
Theorem 2.2) - justified by the $L^{2}$-integrability of $\Delta_{h} K$, the boundedness of $\psi, f$ and $X(\omega)$ - yields

$$
\begin{aligned}
\int_{0}^{t} \psi^{h}(T-s) d Z_{s}= & \int_{0}^{t}\left(\int_{0}^{T-s} F(u, \psi(u)) \Delta_{h} K(T-s-u) d u\right) d Z_{s} \\
= & \int_{0}^{T} F(u, \psi(u))\left(\int_{0}^{t \wedge(T-u)} \Delta_{h} K(T-u-s) d Z_{s}\right) d u \\
= & \int_{0}^{T-t} F(u, \psi(u))\left(\int_{0}^{t} \Delta_{h} K(T-u-s) d Z_{s}\right) d u \\
& +\int_{T-t}^{T} F(u, \psi(u))\left(\int_{0}^{T-u} \Delta_{h} K(T-u-s) d Z_{s}\right) d u \\
= & \int_{t}^{T} F(T-s, \psi(T-s)) g_{t}^{h}(s) d s \\
& +\int_{0}^{t} F(T-s, \psi(T-s)) d\left(X_{s}^{h}-G_{0}(s)\right) \\
= & \int_{t}^{T} F(T-s, \psi(T-s)) d\left(G_{t}^{h}(s)-G_{0}(s)\right) \\
& +\int_{0}^{t} F(T-s, \psi(T-s)) d\left(X_{s}^{h}-G_{0}(s)\right)
\end{aligned}
$$

where we used in the fourth identity that $\left(X_{s}^{h}-G_{0}(s)\right)=\int_{0}^{s}\left(\int_{0}^{r} \Delta_{h} K(r-u) d Z_{u}\right) d r$, due to Lemma 2.1 since $\Delta_{h} K \in L_{\text {loc }}^{2}$, for $h>0$. Recalling (6.8)-(6.9) and sending $h \rightarrow 0$ in the previous identity yields, by invoking the dominated convergence for the left-hand side and Helly's second theorem on $\left(X^{h}, G_{t}^{h}\right)$ for the right-hand side (see Natanson (2016, Theorem 7.3)), we obtain that

$$
\begin{gathered}
\int_{0}^{t} \psi(T-s) d Z_{s}=\int_{t}^{T} F(T-s, \psi(T-s)) d G_{t}(s)+\int_{0}^{t} F(T-s, \psi(T-s)) d X_{s} \\
-\int_{0}^{T} F(T-s, \psi(T-s)) d G_{0}(s) .
\end{gathered}
$$

Using (2.5), we can rewrite $\beta^{T}$ given in (6.2) as

$$
\beta_{s}^{T}=f_{0}(T-s)+b \psi(T-s)-F(T-s, \psi(T-s)) .
$$

This shows that $V^{T}$ given by (6.1) can be re-expressed in the form

$$
\begin{aligned}
V_{t}^{T}=V_{0}^{T}+ & \int_{0}^{t} f_{0}(T-s) d X_{s}+\int_{0}^{t} \psi(T-s) d Z_{s} \\
& -\int_{0}^{t} F(T-s, \psi(T-s)) d X_{s}+\int_{0}^{t} f_{1}(T-s) d M_{s}^{c}+\int_{0}^{t} f_{2}(T-s) d M_{s}^{d} .
\end{aligned}
$$


Plugging (6.10) in the previous expression and recalling (6.3) yields (6.7).

Combining the two previous Lemma, we prove Theorem 2.2.

Proof of Theorem 2.2. Throughout the proof we fix $\psi \in C([0, T], \mathbb{C})$ a solution to the Riccati equation (2.4)-(2.5) satisfying (2.8). We first prove (2.9), and then deduce the weak uniqueness statement. Let $X$ be a non-decreasing nonnegative continuous weak solution to (1.1) for the input $\left(G_{0}, K, b, c, \nu\right)$. By Lemma $6.1 \exp \left(V^{T}\right)$ is a true martingale on $[0, T]$. Its terminal value can be computed using (6.7):

$$
V_{T}^{T}=\int_{0}^{T} f_{0}(T-s) d X_{s}+\int_{0}^{T} f_{1}(T-s) d M_{s}^{c}+\int_{0}^{T} f_{2}(T-s) d M_{s}^{d} .
$$

Whence, by the martingality property, we have that

$$
\mathbb{E}\left[\exp \left(V_{T}^{T}\right) \mid \mathcal{F}_{t}\right]=\exp \left(V_{t}^{T}\right)
$$

for all $t \leq T$. This proves (2.9). To argue uniqueness, we first observe that $V_{0}^{T}$ given in (6.3) does not depend on the process $X$, but only depends on $G_{0}, K$ and $\psi$. We let $Y$ denote another non-decreasing nonnegative continuous weak solution to (1.1) for the same inputs $\left(G_{0}, K, b, c, \nu\right)$ and we set $f_{1}=f_{2} \equiv 0$. Then, (6.11) holds for $Y$ with the same function $\psi$, so that evaluating the expression at $t=0$ gives

$$
\mathbb{E}\left[\exp \left(\int_{0}^{T} f_{0}(T-s) d Y_{s}\right)\right]=\exp \left(V_{0}^{T}\right)=\mathbb{E}\left[\exp \left(\int_{0}^{T} f_{0}(T-s) d X_{s}\right)\right],
$$

for any continuous function $f_{0}:[0, T] \mapsto i \mathbb{R}$. This yields that the finite-dimensional marginals $\left(X_{t_{1}}, \ldots, X_{t_{m}}\right)$ and $\left(Y_{t_{1}}, \ldots, Y_{t_{m}}\right)$ are equal for any $m$, which proves weak uniqueness.

\section{Application: Hyper-rough Volterra Heston models with jumps}

In this section, we apply our main results to a class of hyper-rough Volterra Heston models with jumps. We fix $\left(G_{0}, K, b, c, \nu\right)$ as in Theorem 2.13 and we let $X$ denote the unique non-decreasing nonnegative continuous weak solution to (1.1) with $Z$ the semimartingale with characteristics (1.2) given by Theorem 2.13. We recall the martingales $M^{c}$ and $M^{d}$ that appear in the decomposition (2.1) of $Z$. After a possible extension of the filtered probability space, we let $M^{c, \perp}$ denote a continuous martingale independent of $M^{c}$, such 
that $\left\langle M^{c, \perp}\right\rangle=X$ and we set $M^{S}=\frac{\rho}{\sqrt{c}} M^{c}+\sqrt{1-\rho^{2}} M^{c, \perp}$ for some $\rho \in[-1,1]$. We consider the following model for the log-price $S$

$$
\begin{aligned}
\log S_{t} & =\log S_{0}-\frac{1}{2} X_{t}+M_{t}^{S}, \quad S_{0}>0, \\
X_{t} & =G_{0}(t)+\int_{0}^{t} K(t-s) Z_{s} d s
\end{aligned}
$$

where we recall the characteristics of $Z$ are $(b X, c X, \nu(d \zeta) X)$. For instance, if $c=0$, then $X$ can be interpreted as the 'integrated intensity' of a self-exiciting jump process, e.g. a Hawkes process, recall Example 2.3; if $\nu=0$, then $Z$ is continuous and $X$ can be seen as a hyper-rough process, see Remark 7.2 below.

The chief example we have in mind for $\left(K, G_{0}\right)$ for applications is the following:

Example 7.1. $\quad$ - $K$ is proportional to the fractional kernel:

$$
K(t)=K_{1}(t) K_{H}(t)
$$

where

$$
K_{H}(t)=\frac{t^{H-1 / 2}}{\Gamma(H+1 / 2)}, \quad t>0,
$$

for some $H \in(-1 / 2,1 / 2]$ and $K_{1}$ is a completely monotone kernel on $[0, \infty)$, e.g. $K_{1} \equiv 1$ or $K_{1}(t)=e^{-\eta t}$, for some $\eta>0$. Under such specification, the assumptions of Theorem 2.13 needed on the kernel are satisfied due to Example 2.4.

- $G_{0}$ is absolutely continuous:

$$
G_{0}(t)=\int_{0}^{t} g_{0}(s) d s, \quad t \geq 0, \quad \text { for some } g_{0} \in \mathcal{G}_{K},
$$

as in Example 2.12, recall Remark 2.14.

The following remark shows that $X$ can be thought of as the 'integrated variance' process in the absence of jumps.

Remark 7.2. Assume that $\nu=0$ and $K \in L_{l o c}^{2}$ (e.g. $H>0$ in the specification of Example (7.1)). It follows from Lemma 2.1 that $X_{t}=\int_{0}^{t} V_{s} d s$ where $(S, V)$ is a rough Volterra Heston model in the terminology of Abi Jaber et al. (2019b, Section 7); El Euch and Rosenbaum (2019) satisfying

$$
\begin{aligned}
d \log S_{t} & =-\frac{1}{2} V_{t} d t+\sqrt{V_{t}} d \widetilde{B}_{t}, \quad S_{0}>0 \\
V_{t} & =g_{0}(t)+\int_{0}^{t} K(t-s) b V_{s} d s+\int_{0}^{t} K(t-s) \sqrt{c V_{s}} d \widetilde{W}_{s}
\end{aligned}
$$


for some Brownian motions $\tilde{B}$ and $\tilde{W}$ obtained from standard martingale representation theorems on a possible extension of the probability space, see for instance Revuz and Yor (2013, Proposition V.3.8). For the fractional kernel with $H \in(0,1 / 2)$, the sample paths of $V$ are Hölder continuous of any order strictly less than $H$ and the process $V$ is said to be 'rough'.

If $K_{H}$ is no longer in $L_{\text {loc }}^{2}$ (e.g. $H<0$ in the specification of Example (7.1)), not only Fubini's interchange breaks down, but it can also be shown that $X$ is nowhere differentiable almost surely, see Jusselin and Rosenbaum (2018, Proposition 4.6). In this case, one cannot really make sense of the spot variance $V$ and is stuck with the 'integrated variance' formulation (7.2), justifying the appellation hyper-rough for such equations.

We are now in place to provide the joint Fourier-Laplace transform of $(\log S, X)$ in (7.1)-(7.2).

Theorem 7.3. Let $\left(K, G_{0}\right)$ be as in Theorem 2.13 and $h_{0}, h_{1}: \mathbb{R}_{+} \mapsto \mathbb{C}$ be continuous functions such that

$$
\Re\left(h_{0}\right) \leq 0 \quad \text { and } \quad 0 \leq \Re\left(h_{1}\right) \leq 1 .
$$

The joint Fourier-Laplace transform of $(X, \log S)$ in (7.1)-(7.2) is given by

$\mathbb{E}\left[\exp \left(\int_{t}^{T} h_{0}(T-u) d X_{u}+\int_{t}^{T} h_{1}(T-u) d \log S_{u}\right) \mid \mathcal{F}_{t}\right]=\exp \left(\int_{t}^{T} F(s, \psi(T-s)) d G_{t}(s)\right)$

for all $t \leq T$, where $G_{t}$ is given by (2.6) and $\psi$ solves the Riccati-Volterra equation

$$
\begin{aligned}
\psi(t)= & \int_{0}^{t} K(t-s) F(s, \psi(s)) d s, \quad t \geq 0, \\
F(s, u)= & h_{0}(s)+\frac{1}{2}\left(h_{1}^{2}(s)-h_{1}(s)\right)+\left(b+\rho \sqrt{c} h_{1}(s)\right) u+\frac{c}{2} u^{2} \\
& +\int_{\mathbb{R}_{+}}\left(e^{u \zeta}-1-u \zeta\right) \nu(d \zeta)
\end{aligned}
$$

Proof. It suffices to prove that the Fourier-Laplace transform

$$
L_{t}=\mathbb{E}\left[\exp \left(\int_{t}^{T} h_{0}(T-u) d X_{u}+\int_{t}^{T} h_{1}(T-u) d \log S_{u}\right) \mid \mathcal{F}_{t}\right]
$$

can be written as

$$
L_{t}=\mathbb{E}\left[\exp \left(\int_{t}^{T} f_{0}(T-s) d X_{s}+\int_{t}^{T} f_{1}(t-s) d M_{s}^{c}\right) \mid \mathcal{F}_{t}\right]
$$

where

$$
f_{0}(t)=h_{0}(t)+\frac{1}{2}\left(h_{1}^{2}(t)-h_{1}(t)\right)-\frac{1}{2} \rho^{2} h_{1}^{2}(t) \quad \text { and } \quad f_{1}(t)=\frac{\rho}{\sqrt{c}} h_{1}(t) .
$$


Indeed, if this the case, the Riccati-Volterra equations (2.4)-(2.5) reduce to (7.3)-(7.4) and the claimed expression for the Fourier-Laplace transform together with the existence of the corresponding solution $\psi$ follow from Theorem 2.13 (with $f_{2} \equiv 0$ ), since

$$
\Re\left(f_{0}\right)+\frac{c}{2} \Re\left(f_{1}\right)^{2}=\Re\left(h_{0}\right)+\frac{1}{2}\left(\Re\left(h_{1}\right)^{2}-\Re\left(h_{1}\right)-\Im\left(h_{1}\right)^{2}\right) \leq 0,
$$

since $\Re\left(f_{0}\right) \leq 0$ and $\Re\left(h_{1}\right) \in[0,1]$. It remains to prove (7.6) by means of a projection argument. For this, we fix $t \leq T$ and we write the variation of (7.1) between $t$ and $T$, recall that $M^{S}=\frac{\rho}{\sqrt{c}} M^{c}+\sqrt{1-\rho^{2}} M^{c, \perp}$, to get

$$
d \log S_{u}=-\frac{1}{2} d X_{u}+\rho d M_{u}^{c}+\sqrt{1-\rho^{2}} d M_{u}^{c, \perp} .
$$

We then observe that

$$
\begin{aligned}
M_{t}: & =\mathbb{E}\left[\exp \left(\sqrt{1-\rho^{2}} \int_{t}^{T} h_{1}(T-s) d M_{s}^{c, \perp}\right) \mid \mathcal{F}_{t} \vee \mathcal{F}^{X}\right] \\
& =\exp \left(\frac{\left(1-\rho^{2}\right)}{2} \int_{t}^{T} h_{1}(T-s)^{2} d X_{s}\right)
\end{aligned}
$$

so that, using successively (7.7), the tower property of the conditional expectation and the fact that $X$ and $Z$ are $\mathcal{F}^{X}$-measurable, $L_{t}$ given by (7.5) satisfies

$$
\begin{aligned}
L_{t} & =\mathbb{E}\left[\exp \left(\int_{t}^{T} h_{0}(T-u) d X_{u}+\int_{t}^{T} h_{1}(T-u) d \log S_{u}\right) \mid \mathcal{F}_{t}\right] \\
& =\mathbb{E}\left[\mathbb{E}\left[\exp \left(\int_{t}^{T} h_{0}(T-u) d X_{u}+\int_{t}^{T} h_{1}(T-u) d \log S_{u}\right) \mid \mathcal{F}_{t} \vee \mathcal{F}^{X}\right] \mid \mathcal{F}_{t}\right] \\
& =\mathbb{E}\left[\exp \left(\int_{t}^{T}\left(h_{0}-\frac{1}{2} h_{1}\right)(T-s) d X_{s}+\int_{t}^{T} \frac{\rho}{\sqrt{c}} h_{1}(T-s) d M_{u}^{c}\right) M_{t} \mid \mathcal{F}_{t}\right]
\end{aligned}
$$

leading to (7.6) due to (7.8). This ends the proof.

In particular, we consider the specification of $G_{0}$ as in Example 7.1, and set

$$
h_{0}(t) \equiv u_{0} \quad \text { and } \quad h_{1}(t) \equiv u_{1}, \quad \text { with } \quad \Re\left(u_{0}\right) \leq 0, \quad \Re\left(u_{1}\right) \in[0,1] .
$$

For $t=0$ and $S_{0}=1$, we have $X_{0}=0, \log S_{0}=0$ and $d G_{0}(s)=g_{0}(s) d s$ so that the unconditional Fourier-Laplace transform reads

$$
\mathbb{E}\left[\exp \left(u_{0} X_{T}+u_{1} \log S_{T}\right)\right]=\exp \left(\int_{0}^{T} F\left(u_{1}, u_{2}, \psi(T-s)\right) g_{0}(s) d s\right),
$$


with

$$
\begin{aligned}
\psi(t) & =\int_{0}^{t} K(t-s) F\left(u_{0}, u_{1}, \psi(s)\right) d s \\
F\left(u_{0}, u_{1}, u_{2}\right) & =u_{0}+\frac{1}{2}\left(u_{1}^{2}-u_{1}\right)+\left(b+\rho \sqrt{c} u_{1} u_{2}\right)+\frac{c}{2} u_{2}^{2}+\int_{\mathbb{R}_{+}}\left(e^{u_{2} \zeta}-1-u_{2} \zeta\right) \nu(d \zeta) .
\end{aligned}
$$

If in addition $g_{0}(t)=x_{0}+\theta \int_{0}^{t} K(s) d s$, for some $x_{0}, \theta \geq 0$ (recall Example 2.12), then, Fubini's theorem leads to

$$
\int_{0}^{T} F\left(u_{0}, u_{1}, \psi(T-s)\right) g_{0}(s) d s=x_{0} \int_{0}^{T} F\left(u_{0}, u_{1}, \psi(s)\right) d s+\theta \int_{0}^{T} \psi(s) d s
$$

so that

$$
\mathbb{E}\left[\exp \left(u_{0} X_{T}+u_{1} \log S_{T}\right)\right]=\exp \left(x_{0} \int_{0}^{T} F\left(u_{0}, u_{1}, \psi(s)\right) d s+\theta \int_{0}^{T} \psi(s) d s\right) .
$$

Remark 7.4. Using Theorem 2.8, one can prove the convergence of the multifactor Markovian approximations designed in Abi Jaber (2019); Abi Jaber and El Euch (2019b) towards the hyper-rough Heston model, where the kernel $K_{H}$ is approximated by a suitable weighted sum of exponentials $K^{n}(t)=\sum_{i=1}^{n} c_{i}^{n} e^{-\gamma_{i}^{n} t}$. These approximations are therefore still valid for non-positive values of the Hurst index $H \in(-1 / 2,0]$, which would allow the simulation of the process $X$ and the numerical approximation of the Riccati-Volterra equations, we refer to the aforementioned articles for more details.

\section{A Catalytic super-Brownian motion and its local occupa- tion time}

In this section, we sketch a rigorous derivation of equation (1.7) satisfied by the local occupation time $X$ given by (1.5) formally derived in the introduction. We will make use of the notation $\langle\mu, \phi\rangle$ to denote the quantity $\int_{\mathbb{R}} \mu(d x) \phi(x)$.

We recall that the super-Brownian motion with a single point catalyst $\bar{Y}$ solves the following martingale problem

$$
\left\langle\bar{Y}_{t}, \phi\right\rangle=\left\langle\bar{Y}_{0}, \phi\right\rangle+\frac{1}{2} \int_{0}^{t}\left\langle\bar{Y}_{s}, \Delta \phi\right\rangle d s+\phi(0) Z_{t}
$$

where $\Delta=\partial^{2} / \partial x^{2}, \phi$ is a suitable test function and $Z$ is a continuous martingale with quadratic variation

$$
\langle Z\rangle_{t}=X_{t}
$$


where $X$ is the local occupation time defined by (1.5), see Dawson and Fleischmann (1994, Theorem 1.2.7).

In order to make the link with stochastic Volterra equations, we first reformulate the martingale problem in its 'mild form'.

Lemma A.1. Assume that $\psi \in C^{2}(\mathbb{R}, \mathbb{R})$ has a Gaussian decay, that is $\sup _{x \in \mathbb{R}}|\psi(x)| e^{c z^{2}}<$ $\infty$, for some constant $c$. Then,

$$
\left\langle\bar{Y}_{t}, \psi\right\rangle=\left\langle S_{t} \bar{Y}_{0}, \psi\right\rangle+\int_{0}^{t}\left(S_{t-s} \psi\right)(0) d Z_{s}
$$

where

$$
\left(S_{t} \mu\right)(x)=\int_{\mathbb{R}} p_{t}(x-y) \mu(d y) \quad \text { and } \quad p_{t}(x)=\frac{1}{\sqrt{2 \pi t}} \exp \left(-\frac{x^{2}}{2 t}\right), \quad x \geq 0 .
$$

Sketch of proof. Let $\xi: \mathbb{R}_{+} \rightarrow \mathbb{R}$ be a differentiable function and set $\phi_{t}(x)=\xi(t) \phi^{0}(x)$ for some $C^{2}$ function $\phi^{0}$ having a Gaussian decay. An application of Itô's Lemma gives

$$
\begin{aligned}
d\left\langle\bar{Y}_{t}, \phi^{0}\right\rangle \xi(t) & =\xi(t) d\left\langle\bar{Y}_{t}, \psi\right\rangle+\left\langle\bar{Y}_{t}, \phi^{0}\right\rangle \xi^{\prime}(t) d t \\
& =\left\langle\bar{Y}_{t}, \frac{1}{2} \Delta \phi_{t}+\partial_{t} \phi_{t}\right\rangle d t+\phi_{t}(0) d Z_{t}
\end{aligned}
$$

Thus,

$$
\left\langle\bar{Y}_{t}, \phi_{t}\right\rangle=\left\langle\bar{Y}_{0}, \phi_{0}\right\rangle+\int_{0}^{t}\left\langle\bar{Y}_{s}, \frac{1}{2} \Delta \phi_{s}+\partial_{t} \phi_{s}\right\rangle d s+\int_{0}^{t} \phi_{s}(0) d Z_{s} .
$$

Fix $t \geq 0$ and consider $\phi_{s}=S_{t-s} \psi$ for all $s \in[0, t]$. Noticing that $\phi_{t}=\psi$ and $\partial \phi_{s}=-\frac{1}{2} \Delta \phi_{s}$, the claimed identity follows from (A.1) with this specific test function combined with a density argument.

For each $\varepsilon>0$, let $p_{\varepsilon}: x \rightarrow(2 \pi \varepsilon)^{-1 / 2} \exp \left(-x^{2} /(2 \varepsilon)\right)$ be Gaussian density approximations of the dirac mass at 0 . It follows from Lemma A.1 that

$$
\left\langle\bar{Y}_{t}, p^{\varepsilon}\right\rangle=\left\langle S_{t} \bar{Y}_{0}, p^{\varepsilon}\right\rangle+\int_{0}^{t}\left(S_{t-s} p^{\varepsilon}\right)(0) d Z_{s}
$$

Integrating both sides with respect to time and invoking stochastic Fubini's theorem, see Lemma 2.1, leads to

$$
\int_{0}^{t}\left\langle\bar{Y}_{s}, p^{\varepsilon}\right\rangle d s=\int_{0}^{t}\left\langle S_{s} \bar{Y}_{0}, p^{\varepsilon}\right\rangle d s+\int_{0}^{t}\left(S_{t-s} p^{\varepsilon}\right)(0) Z_{s} d s .
$$

Sending $\varepsilon \rightarrow 0$ yields

$$
X_{t}=\lim _{\varepsilon \rightarrow 0} \int_{0}^{t}\left\langle\bar{Y}_{s}, p^{\varepsilon}\right\rangle d s=\int_{0}^{t}\left(S_{s} \bar{Y}_{0}\right)(0) d s+\int_{0}^{t} p_{t-s}(0) Z_{s} d s
$$

showing that $X$ solves (1.7) with the function $g_{0}(t)=\left(S_{t} \bar{Y}_{0}\right)(0)$. 


\section{References}

Abi Jaber, E. (2019). Lifting the Heston model. Quantitative Finance, 19(12):1995-2013.

Abi Jaber, E., Cuchiero, C., Larsson, M., and Pulido, S. (2019a). A weak solution theory for stochastic Volterra equations of convolution type. arXiv preprint arXiv:1909.01166.

Abi Jaber, E. and El Euch, O. (2019a). Markovian structure of the Volterra Heston model. Statistics 83 Probability Letters, 149:63-72.

Abi Jaber, E. and El Euch, O. (2019b). Multifactor approximation of rough volatility models. SIAM Journal on Financial Mathematics, 10(2):309-349.

Abi Jaber, E., Larsson, M., Pulido, S., et al. (2019b). Affine Volterra processes. The Annals of Applied Probability, 29(5):3155-3200.

Billingsley, P. (1999). Convergence of Probability Measures (2nd ed.). John Wiley \& Sons, Inc.

Brezis, H. (2010). Functional analysis, Sobolev spaces and partial differential equations. Springer Science \& Business Media.

Cuchiero, C. and Teichmann, J. (2020). Generalized Feller processes and Markovian lifts of stochastic Volterra processes: the affine case. Journal of Evolution Equations, pages $1-48$.

Dawson, D. A. and Fleischmann, K. (1991). Critical branching in a highly fluctuating random medium. Probability theory and related fields, 90(2):241-274.

Dawson, D. A. and Fleischmann, K. (1994). A super-Brownian motion with a single point catalyst. Stochastic Processes and their Applications, 49(1):3-40.

Dawson, D. A., Fleischmann, K., Li, Y., and Mueller, C. (1995). Singularity of superBrownian local time at a point catalyst. The Annals of Probability, pages 37-55.

El Euch, O. and Rosenbaum, M. (2019). The characteristic function of rough Heston models. Mathematical Finance, 29(1):3-38.

Etheridge, A. (2000). An introduction to superprocesses. Number 20. American Mathematical Soc.

Fleischmann, K. and Le Gall, J.-F. (1995). A new approach to the single point catalytic super-Brownian motion. Probability theory and related fields, 102(1):63-82.

Gatheral, J., Jaisson, T., and Rosenbaum, M. (2018). Volatility is rough. Quantitative Finance, 18(6):933-949. 
Gripenberg, G., Londen, S.-O., and Staffans, O. (1990). Volterra integral and functional equations, volume 34. Cambridge University Press.

Hawkes, A. G. and Oakes, D. (1974). A cluster process representation of a self-exciting process. Journal of Applied Probability, 11(3):493-503.

Jacod, J. and Shiryaev, A. N. (2003). Limit Theorems for Stochastic Processes (2nd ed.). Springer Berlin Heidelberg.

Jarrow, R. A. (2018). Continuous-Time Asset Pricing Theory. Springer.

Jusselin, P. and Rosenbaum, M. (2018). No-arbitrage implies power-law market impact and rough volatility. e-print arXiv:1805.07134.

Kallsen, J. (2006). A didactic note on affine stochastic volatility models. In From stochastic calculus to mathematical finance, pages 343-368. Springer.

Lépingle, D. and Mémin, J. (1978). Sur l'intégrabilité uniforme des martingales exponentielles. Zeitschrift für Wahrscheinlichkeitstheorie und verwandte Gebiete, 42(3):175-203.

Mandelbrot, B. B. and Van Ness, J. W. (1968). Fractional brownian motions, fractional noises and applications. SIAM review, 10(4):422-437.

Mytnik, L. and Salisbury, T. S. (2015). Uniqueness for Volterra-type stochastic integral equations. arXiv preprint arXiv:1502.05513.

Natanson, I. P. (2016). Theory of functions of a real variable. Courier Dover Publications.

Perkins, E. (2002). Part ii: Dawson-Watanabe superprocesses and measure-valued diffusions. Lectures on probability theory and statistics, pages 125-329.

Revuz, D. and Yor, M. (2013). Continuous martingales and Brownian motion, volume 293. Springer Science \& Business Media.

Veraar, M. (2012). The stochastic Fubini theorem revisited. Stochastics, 84(4):543-551.

Zähle, H. (2005). Space-time regularity of catalytic super-Brownian motion. Mathematische Nachrichten, 278(7-8):942-970. 